Bundesgesundheitsbl 2017 · 60:896-922

DOI 10.1007/s00103-017-2576-z

c) Springer-Verlag GmbH Deutschland 2017
Bekanntmachung des Bundesministeriums für Gesundheit

\section{Format für die Berichterstattung der zuständigen obersten Landesbehörden an das Bundesministerium für Gesundheit/Umweltbundesamt gemäß Richtlinie 98/83/EG und Trinkwasserverordnung}

\section{Ergänzt für die Berichterstattung der Qualität des Trinkwassers hinsichtlich der Parameter Radon, Tritium und Richtdosis}

\section{Einleitung}

Die rechtliche Grundlage des nationalen Berichtsformates bildet $\$ 21$ Absatz 3 der Trinkwasserverordnung (TrinkwV 2001, [1]). Diese Regelung bestimmt, dass die jährlichen Berichte der zuständigen obersten Landesbehörden über die Qualität des Trinkwassers dem von der EU-Kommission festgelegten Format einschließlich der dort genannten Mindestinformationen zu entsprechen haben. Das anzuwendende nationale Berichtsformat wird vom BMG nach Beteiligung der Länder mitgeteilt.

Das BMG veröffentlichte 2013 (wie bereits 2008; [2]) in einer Mitteilung das „Format für die Berichterstattung der zuständigen obersten Landesbehörden an das Bundesministerium für Gesundheit/ Umweltbundesamt gemäß der Richtlinie 98/83/EG (Trinkwasserrichtlinie)“ [3]. Diese Mitteilung, der das „Guidance document on reporting under the Drinking Water Directive 98/83/EC“ (Leitfaden für die Berichterstattung gemäß Trinkwasserrichtlinie 98/83/EG) in der Fassung vom Oktober 2011 [4] zugrunde lag, formulierte die nationalen Anforderungen an
Berichterstattung und Informationsaustausch für die Berichtsjahre ab 2014.

Die Wahrnehmung der Berichtspflichten nach TW-RL verlangte von Bund und Ländern, die Berichterstattung auf ein elektronisches Berichtswesen umzustellen. BMG, UBA und BfG richteten deshalb 2009/2010 im bereits vorhandenen BundLänder-Berichtsportal „WasserBLIcK“ die zentrale Schnittstelle für Trinkwasserdaten bei BfG und UBA ein und schufen damit die informationstechnischen Voraussetzungen, um die Trinkwasserdaten der Länder an den Bund (UBA/BMG) nach Maßgabe des (jeweils aktuellen) Formats für die Berichterstattung zu übermitteln. Auf diesem tabellenorientierten Format basiert der 2012 und 2015 veröffentlichte Bericht des BMG und UBA an die Verbraucherinnen und Verbraucher über die Qualität von Wasser für den menschlichen Gebrauch für den Berichtszeitraum 2008 bis 2010 bzw. 2011 bis 2013.

\section{Berichterstattung gemäß TW-RL}

Die EU-Kommission gibt seit 1995 für WVG ab einer Größe von $1000 \mathrm{~m}^{3}$ pro Tag gelieferten Wassers für den menschlichen Gebrauch oder 5000 versorgten Personen ein Format für die Berichte nach TW-RL vor, dem die Parameterwerte der TWRL zugrunde liegen. Nach TW-RL kann ein Mitgliedstaat zusätzliche Parameter und strengere Parameterwerte festsetzen, wenn es der Schutz der menschlichen Gesundheit im eigenen Hoheitsgebiet erfordert. So legt die TrinkwV 2001 ergänzend für die Parameter Uran, Calcitlöseka-

\begin{tabular}{|ll}
\hline Abkürzungen \\
\hline BfG & Bundesanstalt für Gewässerkunde \\
$B M G$ & Bundesministerium für Gesundheit \\
$B M U B$ & Bundesministerium für Umwelt, \\
& Naturschutz, Bau und Reaktorsicher- \\
& heit \\
EU & Europäische Union \\
ID & Identifikationsbezeichnung \\
TWI & Trinkwasser-Installation (Hausinstal- \\
& lation) \\
NUTS & Nomenclature des unités territoriales \\
& statistiques (dt. Systematik der \\
& Gebietseinheiten für die Statistik) \\
TW-RL & Trinkwasserrichtlinie (Richtlinie \\
& $98 / 83 /$ EG) \\
WISE & Water Information System for Europe \\
UBA & Umweltbundesamt \\
WVG & Wasserversorgungsgebiet(e) \\
\hline
\end{tabular}


pazität und Koloniezahl bei $36^{\circ} \mathrm{C}$ einen Grenzwert bzw. eine Anforderung fest und setzt z.B. für die Parameter Trihalogenmethane und Trübung strengere Maßstäbe als die TW-RL.

Die TrinkwV 2001 verpflichtet in den $\$ \$ 5$ bis 7 zur Einhaltung der Grenzwerte und Anforderungen, wie sie in Anlage 1 bis 3 TrinkwV 2001 festgeschrieben sind. Bei einer Nichteinhaltung eines Parameterwertes nach TW-RL liegt in jedem Fall auch eine Nichteinhaltung des Grenzwertes oder der Anforderung nach TrinkwV 2001 vor; umgekehrt kann ein solcher Rückschluss nicht vorgenommen werden (vgl. auch BMG-Mitteilung 2008, Vorwort; [2]).

Am 22. Oktober 2013 erließ der Rat der Europäischen Union die „Richtlinie 2013/51/EURATOM des Rates zur Festlegung von Anforderungen an den Schutz der Gesundheit der Bevölkerung hinsichtlich radioaktiver Stoffe in Wasser für den menschlichen Gebrauch“. In der nationalen Umsetzung dieser Richtlinie werden mit der Dritten Verordnung zur Änderung der Trinkwasserverordnung die entsprechenden Anforderungen an die Messung und Überwachung der Trinkwasserqualität im Hinblick auf künstliche und natürliche radioaktive Stoffe festgelegt. Die Dritte Verordnung bestimmt Parameterwerte für Radon, Tritium und die Richtdosis (einschließlich der Radonfolgeprodukte Blei-210 und Polonium-210) und formuliert für die Prüfung und Überwachung der Einhaltung dieser Parameterwerte Anforderungen an die Probennahme, Untersuchungsstrategie, Untersuchungsstellen und Untersuchungsverfahren sowie Vorgaben für die Untersuchungshäufigkeiten.

Weiterhin fordert die Richtlinie 2013/51/EURATOM die Information der Bevölkerung über die Qualität des Trinkwassers in angemessener und geeigneter Weise, des Weiteren über vorgesehene Ausnahmen von dieser Richtlinie und ergriffene Maßnahmen zum Schutz der menschlichen Gesundheit. Deshalb sollen die Parameter Tritium, Radon-222 und Richtdosis in den jährlichen Landesberichten nach $\$ 21$ Abs. 3 TrinkwV 2001 berücksichtigt und in einem zu den übrigen Parametern adäquaten Umfang in das nationale Berichtsformat integriert werden. Dies entspricht dem für die anderen Parameter geltenden Maßstab bezüglich Transparenz für die Verbraucherinnen und Verbraucher.

\section{1 Änderungen des EU- Berichtsformates}

Im Jahr 2014 vereinbarte der Komitologieausschuss nach Artikel 12 TW-RL, das „Guidance document on reporting under the Drinking Water Directive 98/83/EC“ vom Oktober 2011 auf der Grundlage der in den Mitgliedstaaten damit gesammelten Erfahrungen zu überarbeiten und betraute mit dieser Aufgabe eine Unterarbeitsgruppe, die im September 2015 eine aktuelle Fassung vorlegte. ${ }^{1}$ Richteten sich die Änderungen in 2011 u. a. auf die Probennahmestellen und -verfahren und auf eine modifizierte Darstellung von Untersuchungsergebnissen bei Nichteinhaltung von Parameterwerten im betreffenden WVG, so orientiert sich das aktualisierte Berichtsformat noch stringenter als bisher an der Tabellenstruktur und enthält darüber hinaus erstmals auch Abfragen zu den sogenannten kleinen $\mathrm{WVG}{ }^{2}$ über die die EU-Kommission in zunehmendem Maße informiert werden möchte.

Die inhaltlichen Neuerungen im Format der Berichterstattung nach TW-RL verlangen, die Vorgaben zur Erfüllung der nationalen Informationspflichten daran anzupassen. Diese Änderungen betreffen vor allem die konsequente Umsetzung eines tabellarischen Berichtsformates. Bisherige Tabellen werden inhaltlich ergänzt, neu gegliedert oder entfallen, neue Tabellen kommen hinzu. Außerdem berücksichtigt das nachstehende (nationale) Format Ergebnisse der Überwachung auf Radioaktivität im Trinkwasser.

\footnotetext{
1 Das aktualisierte "Guidance document on reporting" von September 2015 liegt (bisher) nur in elektronischer Form und in englischer Sprache vor, siehe auch das "Guidance document on reporting" von September 2014 unter: http:// forum.eionet.europa.eu/x_wise-reporting/library/drinking-water-directive-library/dwd-datarequest-2014/dwd_guidance_document_reporting_sep_22_14-1

2 WVG, in denen mindestens $10 \mathrm{~m}^{3}$ Trinkwasser pro Tag verteilt oder mindestens 50 Personen versorgt werden sowie höchstens $1000 \mathrm{~m}^{3}$ Trinkwasser pro Tag verteilt und höchstens 5000 Personen versorgt werden.
}

Die Grundsätze 2-1 bis 2-11 in der BMG-Mitteilung von 2008 [2] bleiben von den Formatänderungen unberührt und unterliegen ggf. der künftigen Fortschreibung der Schnittstelle durch BfG, BMG und UBA in Zusammenarbeit mit den zuständigen Landesbehörden.

Die obersten Landesbehörden berichten dem UBA ab dem Berichtsjahr 2020 nach dem hier vorgegebenen Format in der in $\$ 21$ Absatz 3 TrinkwV 2001 genannten Frist. Über die Parameter der Radioaktivität in Wasserversorgungsgebieten mit bestehenden Wasserversorgungsanlagen berichten die obersten Landesbehörden unter Beachtung des $\$ 14 \mathrm{a}$ Abs. 2 Satz 2 in Verbindung mit der Anlage 3a Teil III ebenfalls ab dem Berichtsjahr 2020. Für neu in Betrieb genommene Wasserversorgungsanlagen ist die Erstuntersuchung innerhalb der auf die Inbetriebnahme folgenden 12 Monate abzuschließen und in den Bericht des darauf folgenden Berichtsjahres aufzunehmen.

Der Bericht ist als XML-Datei über die für Trinkwasser spezifizierte XML-Schnittstelle in die zentrale Datenhaltung des „WasserBLIcK“ bei der BfG einzustellen.

\subsection{Informationen, die dem UBA jährlich vorzulegen sind}

\subsubsection{Informationen zur Einhaltung der Mindestüberwachungshäufigkeit in Wasserversorgungsgebieten}

Diese Informationen sind nach dem Format der - Tab. A-1 bereitzustellen. ${ }^{3}$ Untersuchungen der Parameter Radon und Richtdosis sind zu berücksichtigen, wenn die zuständige Behörde keine anderweitige Feststellung nach $\$ 14 \mathrm{a}$ Abs. 4 TrinkwV 2001 getroffen hat. Untersuchungen des Parameters Tritium sind zu berücksichtigen, wenn die zuständige Behörde diese nach $\$ 14$ a Abs. 1 Satz 5 TrinkwV 2001 angeordnet hat.

\footnotetext{
3 Die Tabellennummerierung folgt dem Guidance document (siehe Fußnote 1). Der hinzugefügte Großbuchstabe dient der besseren Zuordnung der im nationalen Format verwendeten Tabellen. Nachfolgend ist die Tabellennummer A-7 nicht vergeben, da im Guidance document eine Tabellennummer 7 fehlt bzw. gestrichen worden ist, ohne die Tabellenreihung daran anzupassen.
} 


\subsubsection{Informationen über die allgemeinen Rahmenbedingungen zur Trinkwasserversorgung im jeweiligen Land}

Das erforderliche Format ist durch

- Tab. A-2, A-11 und A-11-1 bestimmt.

\subsubsection{Informationen zur Qualität von Wasser für den menschlichen Gebrauch in Wasserversorgungsgebieten auf landesweiter Ebene}

Diese Informationen sind in dem Format gemäß $\bullet$ Tab. A-3 wiederzugeben (Raum für weitere, auch fakultative Angaben zu - Tab. A-3 bietet - Tab. A-3-2)

\subsubsection{Information über die Ergebnisse der Nachforschungen bei Nichteinhaltung des Parameterwertes Clostridium perfringens}

Dafür ist $\bullet$ Tab. A-3-1 heranzuziehen.

\subsubsection{Informationen zu Nichteinhaltungen von Parameterwerten in den betroffenen und in - Tab. A-11 bezeichneten Wasserversorgungsgebieten}

Diese Informationen müssen den Vorgaben gemäß - Tab. A-4, A-4-1, A-5 und A-6 entsprechen. Hier sind auch jene von Artikel 9 TW-RL erfassten zugelassenen Abweichungen im Berichtsjahr zu berücksichtigen, über die das UBA bereits nach $\$ 10$ TrinkwV 2001 von den zuständigen obersten Landesbehörden unterrichtet worden ist (vgl. BMG-Mitteilung 2008, Grundsatz 2-11; [2]). Die Parameter Radon, Tritium und Richtdosis sind hier ebenfalls zu berücksichtigen.

Der Begriff „Nichteinhaltung vom Parameterwert" wird zur besseren Lesbarkeit insbesondere in Übersichten und Überschriften im vorliegenden Dokument sowohl auf die „Parameterwerte“ der Trinkwasserrichtlinie und des Guidance documents (die in der Trinkwasserverordnung als "Grenzwerte“ und "Anforderungen" umgesetzt wurden) angewendet wie auch auf die „Parameterwerte“ der Trinkwasserverordnung, die dort nur für die radioaktivitätsbezogenen Parameter existieren. Im Falle der Letzteren handelt es sich um eine „Überschreitung“ des Pa-

Tab. A-1 Jahresübersicht über $\mathrm{WVG}^{7}$, in denen die Überwachungshäufigkeit nicht eingehalten wird ${ }^{2}$

(Informationen zur Anzahl der durchgeführten Untersuchungen verglichen mit der Anzahl der erforderlichen Untersuchungen)

Landescode $^{6}$

Jahr

\begin{tabular}{|c|c|c|c|c|}
\hline $\begin{array}{l}\text { Bezeich- } \\
\text { nung des } \\
\text { WVG }^{1}\end{array}$ & WVG-ID ${ }^{1}$ & $\begin{array}{l}\text { Betrof- } \\
\text { fener } \\
\text { Parameter }\end{array}$ & $\begin{array}{l}\text { Geforderte Anzahl } \\
\text { der Untersuchun- } \\
\text { gen }\end{array}$ & $\begin{array}{l}\text { Anzahl der durchgeführten } \\
\text { Untersuchungen }\end{array}$ \\
\hline
\end{tabular}

Anmerkungen:

'aus Tab. A-11.

Umfang und Häufigkeit der Untersuchungen bestimmen sich nach TrinkwV 2001, wenn eine Trennung der Häufigkeiten mit Bezug auf die Vorgaben der TW-RL nicht möglich ist, ohne der gleichzeitigen Forderung nach Repräsentativität zuwiderzuhandeln. Die genannte Anzahl der Untersuchungen muss die ausgeschöpften Möglichkeiten der Reduzierung nach TW-RL beinhalten unter Einbeziehung der einschlägigen Bestimmungen der TrinkwV 2001 [§ 19 Abs. 2 Satz 2 Nr. 1 und Anlage 4 Teil I Buchstabe a Satz 2 und 3 und Buchstabe b Satz 1 und 2 TrinkwV 2001 und Hinweise in Anlage 3 Teil I lfd. Nr. 4 TrinkwV 2001 (zu Clostridium perfringens), in Anlage 3 Teil I lfd. Nr. 16 TrinkwV 2001 (zu Oxidierbarkeit)]. Die geforderte Mindestanzahl an Untersuchungen ist in einem Probennahmeplan festzuhalten. Etwaige zusätzliche Untersuchungen, die notwendig werden, um die Einhaltung zugelassener Abweichungen vom Grenzwert nach Art. 9TW-RL bzw. §10 TrinkwV 2001 zu überwachen, sind nicht der geforderten Anzahl der Untersuchungen zuzurechnen, die sich aus Anhang II Teil B Tab. 1 TW-RL ableitet. In die Anzahl der Untersuchungen gehen auch jene Untersuchungen zur Betriebskontrolle nicht ein, die der Betreiber zusätzlich zu der vom Gesundheitsamt geforderten Mindestanzahl an

Proben pro Jahr durchführt.

${ }^{3}$ Dies gilt auch für die Anforderung, den Parameterwert für Nitrit am Ausgang des Wasserwerks sicherzustellen (Anhang I Teil B Anmerkung 5 TW-RL).

${ }^{4}$ In die Anzahl der Untersuchungen können jene nach §19 Abs. 7 TrinkwV 2001 (Überwachung in häuslichen Trinkwasser-Installationen) mit eingehen.

${ }^{5}$ Für die Parameter Radon und Richtdosis gelten die Mindesthäufigkeiten der Untersuchungen nach Anlage $3 a$ Teil III Nr. 1 TrinkwV 2001 in Verbindung mit §14a und §20a Abs. 3 TrinkwV 2001; für den Parameter Tritium gilt ggf. die Anordnung der zuständigen Behörde nach §14a Abs. 1 Satz 5 TrinkwV 2001.

${ }^{6}$ Brandenburg: DEBB, Berlin: DEBE, Baden-Württemberg: DEBW, Bayern: DEBY, Bremen: DEHB, Hamburg: DEHH, Hessen: DEHE, Mecklenburg-Vorpommern: DEMV, Niedersachsen: DENI, Nordrhein-Westfalen: DENW, Rheinland-Pfalz: DERP, Schleswig-Holstein: DESH, Saarland: DESL, Sachsen: DESN, Sachsen-Anhalt: DEST, Thüringen: DETH.

WVG, in denen mehr als $1000 \mathrm{~m}^{3}$ Wasser pro Tag verteilt oder mehr als 5000 Personen versorgt werden.

rameterwertes und nicht um eine „Nichteinhaltung", da diese Parameterwerte der Trinkwasserverordnung Werte darstellen, bei deren Überschreitung die zuständige Behörde prüft, ob das Vorhandensein radioaktiver Stoffe im Trinkwasser ein Risiko für die menschliche Gesundheit darstellt, das ein Handeln erfordert. Die Trinkwasserverordnung verwendet deshalb in Bezug auf radioaktive Stoffe korrekterweise die Formulierung „Überschreitung"von Parameterwerten.

\subsubsection{Informationen über die produktspezifischen Parameter Acrylamid, Epichlorhydrin und Vinylchlorid}

(nach dem Format der • Tab. A-8)

\subsubsection{Informationen über die Qualität von Wasser für den menschlichen Gebrauch an die Öffentlichkeit}

(siehe auch BMG-Mitteilung 2008, 4. Kapitel [2])

Nach Artikel 13 Absatz 1 TW-RL sind in den Mitgliedstaaten Maßnahmen zu ergreifen, die sicherstellen, dass den Verbrauchern und Verbraucherinnen geeignetes und aktuelles Informationsmaterial über die Qualität von Wasser für den menschlichen Gebrauch zur Verfügung steht. Nach dem Format der • Tab. A-9 und A-10 ist $\mathrm{zu}$ dokumentieren, wo und in welcher Art sowohl die Öffentlichkeit als auch die EU-Kommission diese Informationen über die Trinkwasserbeschaffenheit im Land abrufen können. 
Tab. A-2 Allgemeine Informationen über die Rahmenbedingungen der Trinkwasserversorgung in dem Land

(Mit Ausnahme von Frage 1.2 werden nur Informationen über WVG gemeldet, in denen mehr als $1000 \mathrm{~m}^{3}$ Wasser für den menschlichen

Gebrauch pro Tag verteilt oder mehr als 5000 Personen versorgt werden.)

1.1.1 Landescode ${ }^{6}$

1.1.2. Jahr

1.2 Gesamtbevölkerung in Mio.

1.3 Anzahl derWVG

1.4 Gesamte in den WVG versorgte Bevölkerung ${ }^{2}$

1.5 Gesamtes in den WVG verteiltes Wasservolumen in Mio. $\mathrm{m}^{3} / \mathrm{Jahr}$

1.6 Für 1.5 genutzte Wasserressourcen (=100\%)

1.6.1 Anteil von 1.6 an Grundwasser ${ }^{3}$ in Prozent

1.6.2 Anteil von 1.6 an Oberflächenwasser ${ }^{4}$ in Prozent

1.6.2.1 Anteil von 1.6 an Binnenwasser in Prozent

1.6.2.2 Anteil von 1.6 an Küsten-, Übergangs-, (Brack)wasser in Prozent

1.6.3 Anteil von 1.6 an Uferfiltrat in Prozent

1.6.4 Anteil von 1.6 an künstlicher Grundwasseranreicherung in Prozent

1.6.5 Anteil von 1.6 an Regenwasser ${ }^{5}$ in Prozent

1.6.6 Anteil von 1.6 an sonstigen Ressourcen in Prozent

1.7 Landesdatenbank zur Qualität von Wasser für den menschlichen Gebrauch

1.7.1 Öffentlicher Zugang zur Datenbank unter folgender Webseiten-Adresse

1.8 Kontaktstelle im Land

1.8.1 Zuständige Behörde

1.8.2 Ansprechpartner

1.8.3 Anschrift

1.8.4 Telefon

1.8.5 Fax

1.8.6 E-Mail

Anmerkungen:

'Die Gesamtbevölkerung in Mio. soll bis zu drei Stellen nach dem Komma angegeben werden.

${ }^{2}$ Die Bevölkerung in 1.4 wird als ganze Zahl (nicht durch Dezimalbezeichnungen abgekürzt) gemeldet.

${ }^{3}$ Grundwasser bedeutet alles unterirdische Wasser in der Sättigungszone, das in unmittelbarer Berührung mit dem Boden oder dem Untergrund steht.

${ }^{4}$ Oberflächenwasser bedeutet Binnenwasser (ohne Grundwasser), Übergangswasser und Küstenwasser (Wasserrahmenrichtlinie 2000/60/EG Art. 2.1).

${ }^{5}$ Regenwasser bedeutet hier nur Regenwasser, das ausschließlich als Ressource für die Trinkwasserversorgung genutzt wird. Hierzu gehört nicht Dachablaufwasser für häusliche Zwecke.

${ }^{6}$ Siehe Tab. A-1 Anm. 6. 


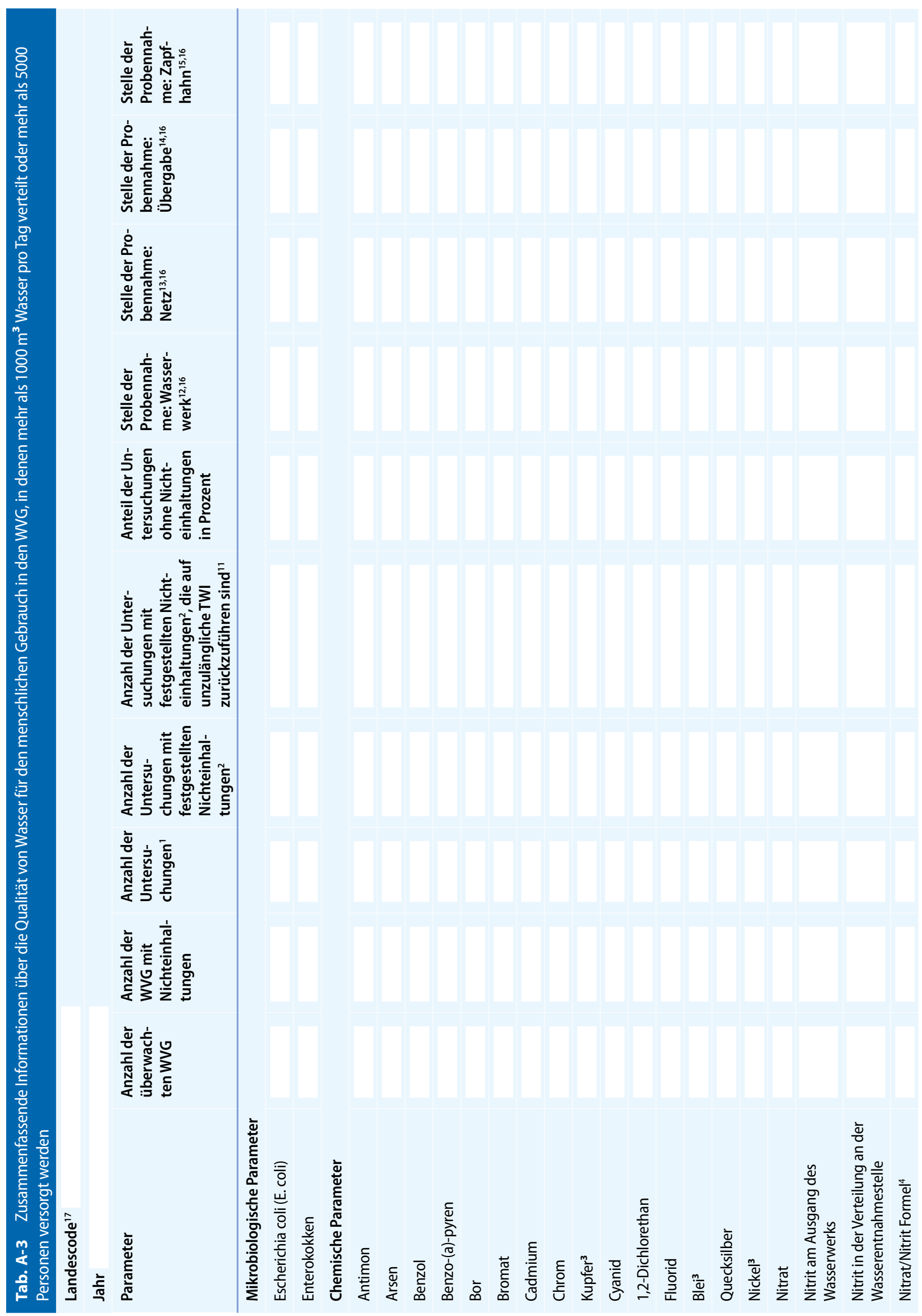




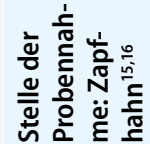

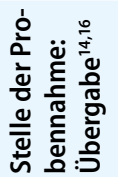

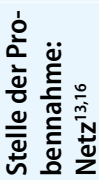

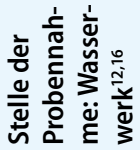

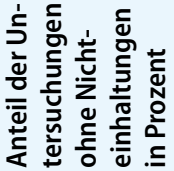

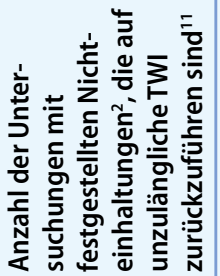

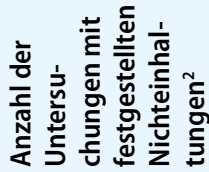

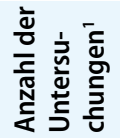

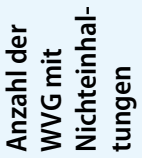

这它
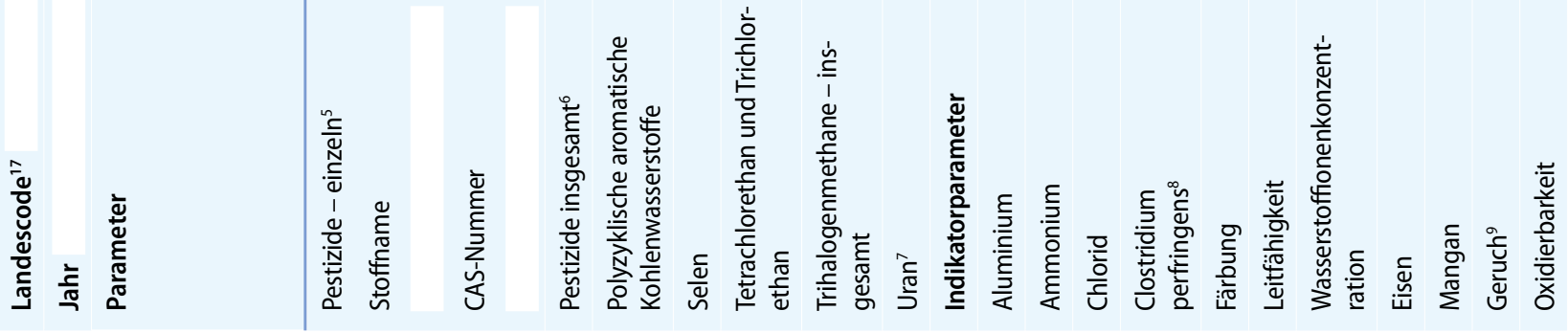


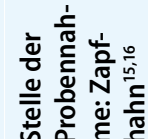

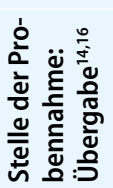

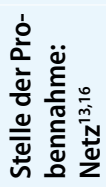

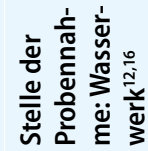

รั ธั่ง ฮ

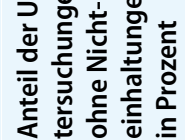

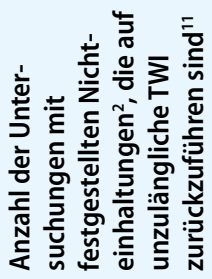

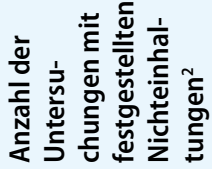

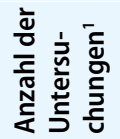

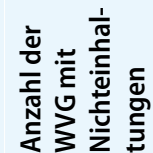

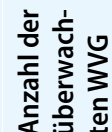

कำ

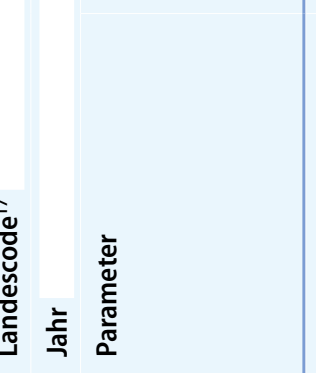

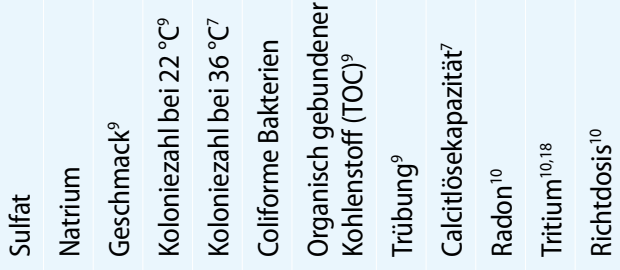




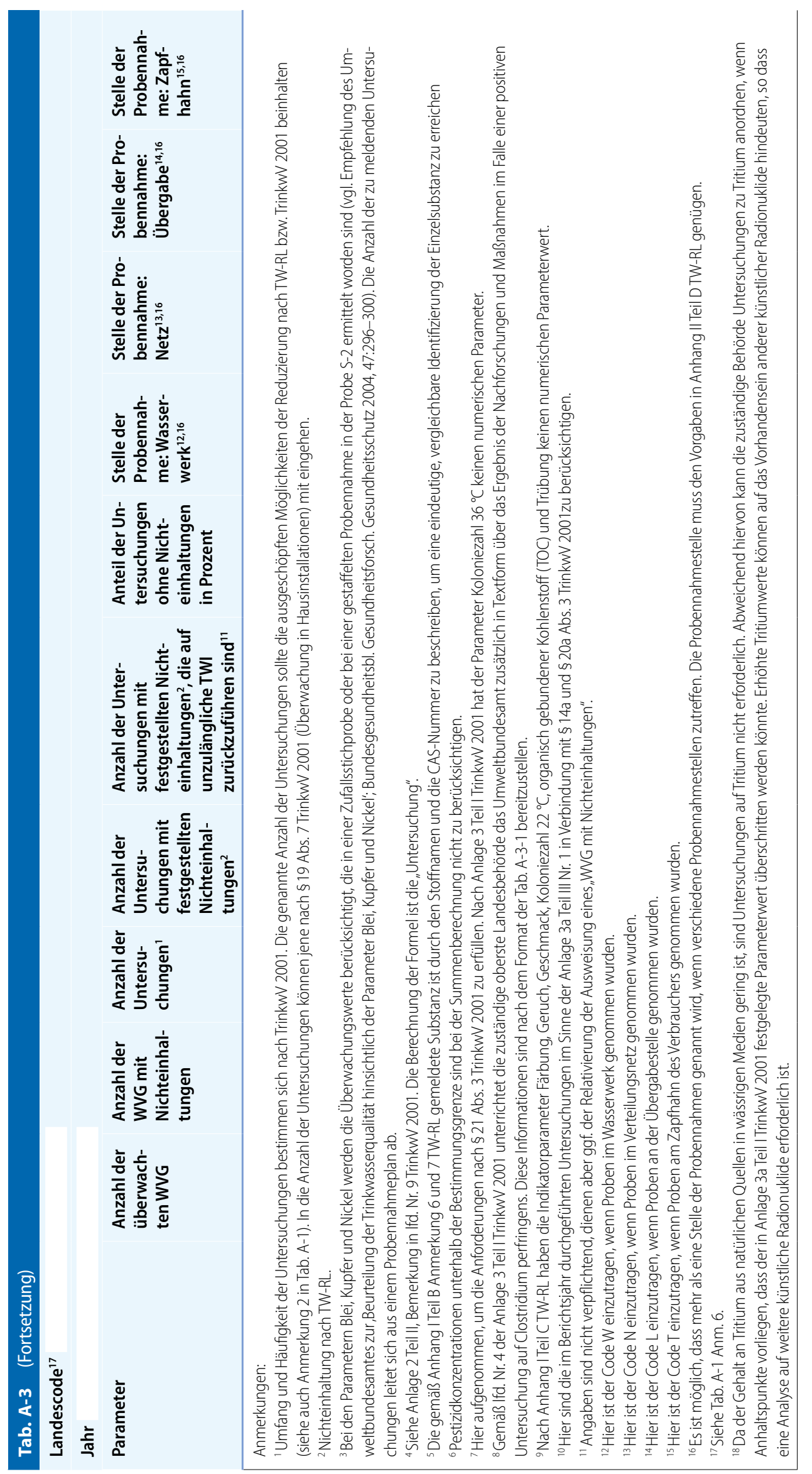


Tab. A-3-1 Information über die Ergebnisse der Nachforschungen bei Nichteinhaltung des Parameterwertes Clostridium perfringens (einschließlich Sporen) im WVG7 gemäß Anlage 3 lfd. Nr. 4 TrinkwV 2001,2

Landescode ${ }^{6}$

Jahr

WVG $^{3}$

WVG-ID

Datum der ersten Feststellung der Nichteinhaltung

Zeitspanne bis zur Beseitigung der Nichteinhaltung (in Tagen)

Festgestellte Nichteinhaltung (Anzahl/100 ml; maximal drei Messwerte)

Zusätzliche Grenzwertüberschreitungen bei coliformen Bakterien, E. coli, Enterokokken

Ja/Nein

Ursache(n) für die Nichteinhaltung

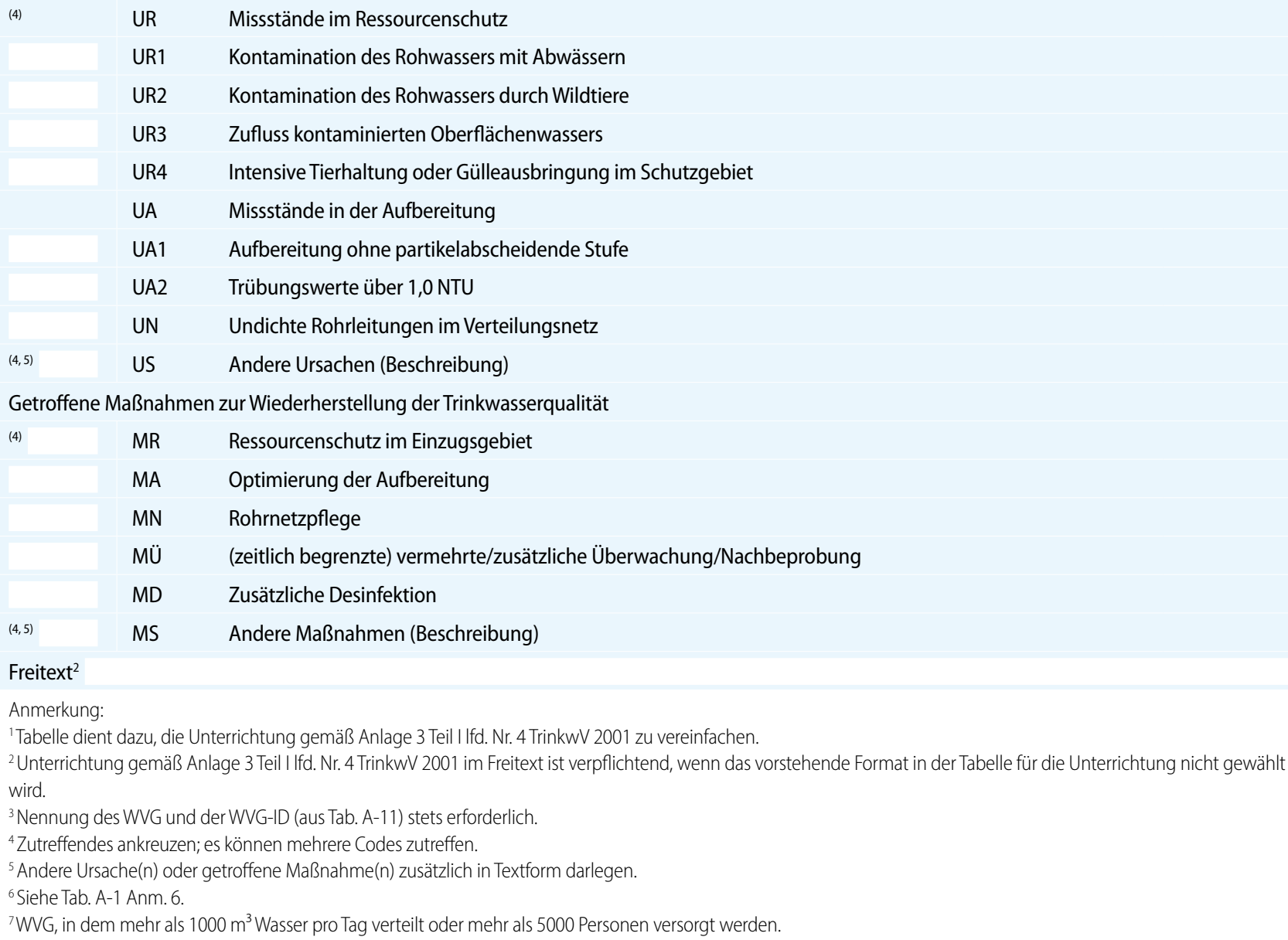

\section{Tab. A-3-2 Weitere Bemerkungen des Landes zu Tab. A-3}

Landescode ${ }^{1}$

Jahr

Freitext

${ }^{1}$ Siehe Tab. A-1 Anm. 6. 
Tab. A-4 Informationen über Nichteinhaltung der Parameterwerte bei Wasser für den menschlichen Gebrauch in WVG, in denen mehr als

$1000 \mathrm{~m}^{3}$ Wasser pro Tag verteilt oder mehr als 5000 Personen versorgt werden

Landescode ${ }^{8}$

Jahr

$\begin{array}{lllllllll}\text { WVG } & \begin{array}{l}\text { Para- } \\ \text { WVG-ID } \\ \text { meter }{ }^{1}\end{array} & \begin{array}{l}\text { Ggf. Aktenzeichen der } \\ \text { zuständigen obersten } \\ \text { Landesbehörde für } \\ \text { die Zulassung einer } \\ \text { Abweichung }\end{array} & \begin{array}{l}\text { Anzahl der } \\ \text { betroffenen } \\ \text { Bevölkerung }\end{array} & \begin{array}{l}\text { Gesamtzahl der } \\ \text { Untersuchungen } \\ \text { (im Berichtsjahr) }\end{array} & \begin{array}{l}\text { Gesamtzahl der Un- } \\ \text { tersuchungen mit } \\ \text { Nichteinhaltung } \\ \text { Max }{ }^{3}\end{array} & \begin{array}{l}\text { Med } \\ \text { total }^{4}\end{array} & \begin{array}{l}\text { Med non- } \\ \text { complient }^{5}\end{array} & \begin{array}{l}\text { NCl_ROW- } \\ \text { ID }^{6,7}\end{array} \\ \end{array}$

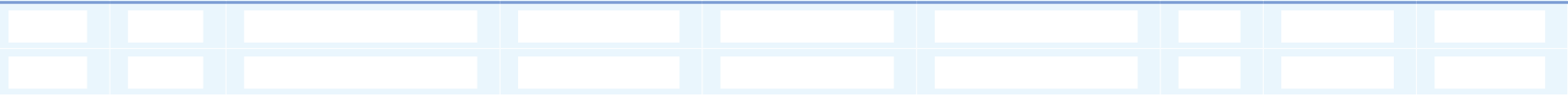

Anmerkung:

${ }^{1}$ Zu berücksichtigen sind Nichteinhaltungen vom Parameterwert jedes Parameters in Anhang I Teil A bis C TW-RL bzw. zugelassene Abweichungen vom Parameterwert jedes Parameters in Anhang I Teil B TW-RL. Hier sind auch Überschreitungen der Parameterwerte für Radon, Tritium und Richtdosis gemäß TrinkwV 2001 zu berücksichtigen, nachfolgende Anmerkungen gelten entsprechend.

${ }^{2}$ Zu berücksichtigen sind alle Ergebnisse, die die Parameterwerte der TW-RL nicht erfüllen, einschließlich jener, die unter eine zugelassene Abweichung nach Art. 9 TW-RL fallen (vgl. Anm. 7).

${ }^{3}$ Der Wert zeigt die Höchstkonzentration des betroffenen Parameters im Trinkwasser des WVG an, bezogen auf das Berichtsjahr oder das separate Ereignis (vgl. Anm. 7).

${ }^{4}$ Medianwert aller Untersuchungsergebnisse des betroffenen Parameters im Berichtsjahr.

${ }^{5}$ Medianwert der Untersuchungsergebnisse, die den Parameterwert der TW-RL nicht erfüllen (vgl. auch Anm. 7).

${ }^{6}$ Hier ist der eindeutige ID-Code der Nichteinhaltung des betroffenen Parameters einzutragen.

${ }^{7}$ Tritt in einem WVG beim gleichen Parameter mehr als ein Ereignis mit Nichteinhaltung auf und lassen sich die Nichteinhaltungen auf verschiedene Ursachen zurückführen, dann werden die Ereignisse in getrennten Zeilen aufgeführt. Jede Zeile ist mit einer eigenen NCI_ROW-ID (in Spalte 11) zu kennzeichnen. Liegen mehr als ein Ereignis

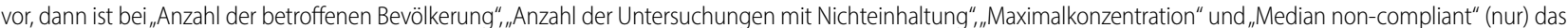
jeweilige Ereignis (nicht das gesamte Berichtsjahr) zu berücksichtigen. Die Benennung der verschiedenen Ursachen ist der Angabe Kombination an Ursachen (mit Code S in Tab. A-5) vorzuziehen. (Zeitlich versetzte) Ereignisse mit gleicher Ursache werden als ein Ereignis im Berichtsjahr betrachtet.

${ }^{8}$ Siehe Tab. A-1 Anm. 6.

${ }^{9}$ Das Az. ist einzutragen, wenn es sich um eine Zulassung/Beantragung einer Abweichung im Sinne von $\S 10$ Abs. 3, 5 oder 6 TrinkwV 2001 handelt.

Tab. A-4-1 Informationen über Einschränkung/Unterbrechung der Bereitstellung von Wasser für den menschlichen Gebrauch wegen Nichteinhaltung der Parameterwerte im WVG

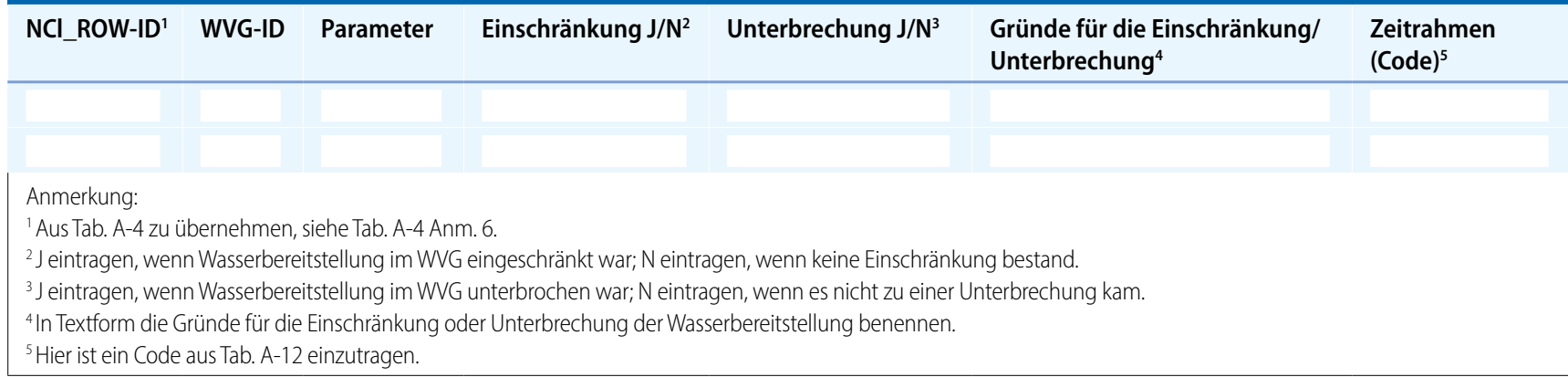

Tab. A-5 Informationen über Ursachen der Nichteinhaltung der Parameterwerte im WVG

\begin{tabular}{|c|c|c|c|c|c|}
\hline NCl_ROW-ID ${ }^{1}$ & WVG-ID & Parameter & Ursache $^{2}$ & Anzahl der Untersuchungen mit Nichteinhaltung ${ }^{3}$ & Remedial-ID 4 \\
\hline \multicolumn{6}{|c|}{ 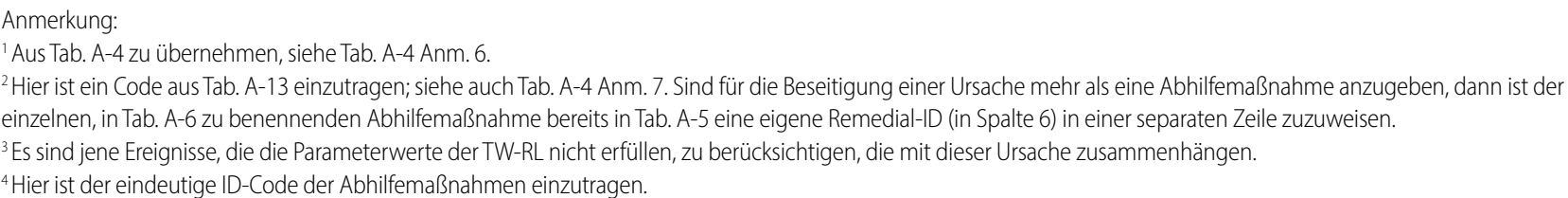 } \\
\hline
\end{tabular}


Tab. A-6 Informationen über Abhilfemaßnahmen bei Nichteinhaltung der Parameterwerte im WVG

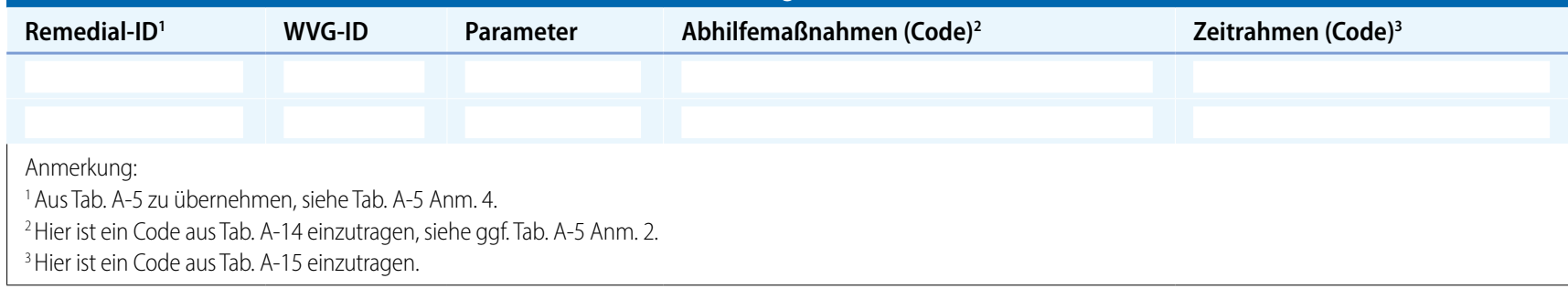

\section{Tab. A-8 Informationen über die produktspezifischen Parameter Acrylamid, Epichlorhydrin und Vinylchlorid}

\section{Landescode ${ }^{2}$}

Jahr

Parameter Erläuterungen, wie die Einhaltung der Anforderungen sichergestellt ist ${ }^{1}$

Acrylamid

Epichlorhydrin

Vinylchlorid

Anmerkung:

${ }^{1}$ Gemäß Anhang I Teil B Anmerkung 1 TW-RL müssen die produktbezogenen Parameter Acrylamid, Epichlorhydrin und Vinylchlorid nicht im Wasser analysiert werden, dennoch muss gemäß den Produktspezifikationen des entsprechenden Polymers in Kontakt mit Trinkwasser nachgewiesen werden, dass der Parameterwert nach TW-RL eingehalten wird. Das Land erläutert, wie es diese Anforderung der TW-RL erfüllt hat, z. B. länderspezifische Regelungen für Bauprodukte in Kontakt mit Trinkwasser. Wird

Trinkwasser auch auf Acrylamid, Epichlorhydrin oder Vinylchlorid chemisch analysiert, dann ist das in dieser Tabelle zu vermerken.

${ }^{2}$ Siehe Tab. A-1 Anm. 6.

Tab. A-9 Art der im Land öffentlich zugänglichen Informationen

\section{Landescode ${ }^{1}$}

Jahr

Art der verfügbaren Informationen

Ja/Nein

Ort der Information ${ }^{2}$

9.1 Landesweite Zusammenfassung über die Einhaltung der TW-RL

9.2 Regionale Zusammenfassung über die Einhaltung der TW-RL

9.3 Zusammenfassung der Einhaltung der TW-RL bezogen auf das WVG

9.4 Einzelne Überwachungsergebnisse für das WVG

9.5 Überwachungsergebnisse für kleine WVG (in denen im Durchschnitt 10 bis $1000 \mathrm{~m}^{3} / \mathrm{Tag}$ verteilt und 50 bis 5000 Personen versorgt werden)

9.6 Überwachungsergebnisse für sehr kleine WVG (in denen im Durchschnitt weniger als $10 \mathrm{~m}^{3} /$ Tag verteilt und weniger als 50 Personen versorgt werden)

9.7 Informationen über Einhaltung strengerer nationaler Standards

9.8 Informationen über Einhaltung zusätzlicher nationaler Standards

9.9 Informationen auf der Ebene eines WVG über Nichteinhaltungen

9.10 Informationen über die Trinkwasserressource(n) im betreffenden WVG

9.11 Sonstiges

Anmerkungen:

1 Siehe Tab. A-1 Anm. 6

${ }^{2}$ Bei dem Ort sollte es sich um die Stelle handeln, an die sich die EU-Kommission wenden kann, um die jeweils aufgeführten Arten von Informationen zu erhalten. 
Tab. A-10 Kommunikation der im Land verfügbaren Informationen über die Qualität von Wasser für den menschlichen Gebrauch

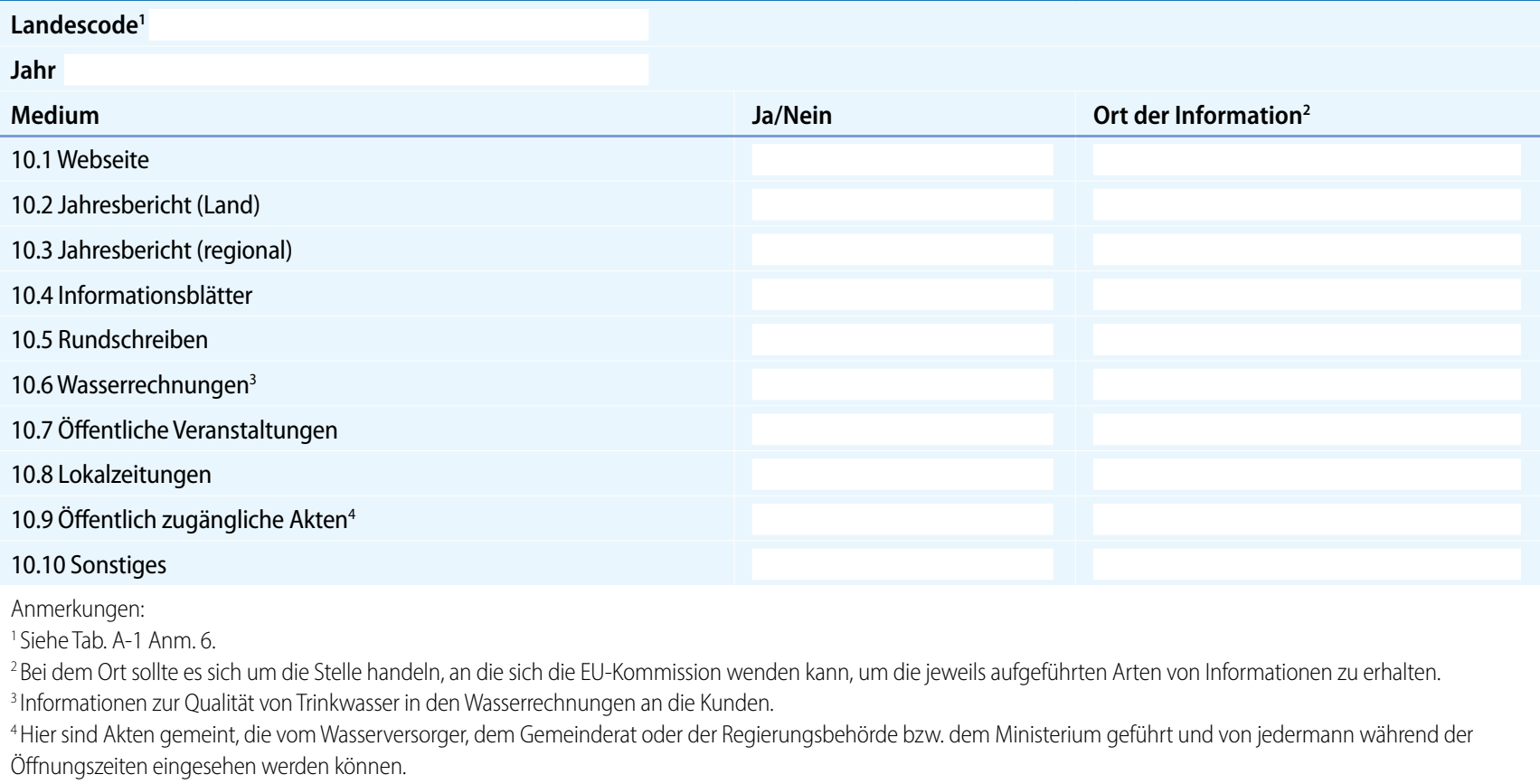

Tab. A-11 Informationen über die Wasserversorgungsgebiete in dem Land

(Informationen über WVG, in denen mehr als $1000 \mathrm{~m}^{3}$ Wasser für den menschlichen Gebrauch pro Tag verteilt oder mehr als 5000 Personen versorgt werden.)

\section{Landescode ${ }^{6}$}

Jahr

\begin{tabular}{|c|c|c|c|c|c|c|c|}
\hline $\begin{array}{l}\text { Bezeichnung } \\
\text { des WVG }{ }^{1}\end{array}$ & WVG-ID² & $\begin{array}{l}\text { LAU2- } \\
\text { Code }^{3}\end{array}$ & $\begin{array}{l}\text { Breitegrad } \\
\text { des WVG } 4\end{array}$ & $\begin{array}{l}\text { Längengrad } \\
\text { des WVG }{ }^{4}\end{array}$ & $\begin{array}{l}\text { Versorgte } \\
\text { Bevölkerung }\end{array}$ & $\begin{array}{l}\text { Abgegebenes Wasservolu- } \\
\text { men in } \mathrm{m}^{3} / \mathrm{Tag}\end{array}$ & RAP $^{7}$ \\
\hline & & & & & & & \\
\hline \multicolumn{8}{|c|}{$\begin{array}{l}\text { 'Bezeichnung oder Name des einzelnen, von der Landesbehörde festgelegten Wasserversorgungsgebiets. } \\
\text { 2Eindeutiger ID-Code des WVG (WVG-ID), Angabe erforderlich. Wird beibehalten, wenn weitere Informationen im Zusammenhang mit dem WVG verlangt sind. } \\
\text { '3NUTS-Codes der Verwaltungseinheiten auf LAU2-Ebene. Es besteht keine Notwendigkeit, die WVG an Verwaltungsgrenzen auszurichten. } \\
{ }^{4} \text { Geographischer Breiten- bzW. Längengrad gemäß World Geodetic System } 1984 \text { (WGS-84) in Dezimalformat bis zu } 6 \text { Dezimalstellen (Beispiel Berlin, Brandenburger Tor: } \\
\text { Breitengrad 52.516275, Längengrad 13.377704). Für die Angabe des Breiten- und Längengrades sollte ein zentraler Punkt innerhalb des WVG (z. B. das [wichtigste] Wasser- } \\
\text { werk) oder der Flächenschwerpunkt der wichtigsten Stadt innerhalb des WVG ausgewählt werden. } \\
{ }^{5} \text { Wird als ganze Zahl (nicht durch Dezimalbezeichnungen abgekürzt) gemeldet. } \\
{ }^{6} \text { Siehe Tab. A-1 Anm. } 6 . \\
\text { 7Hier ankreuzen, wenn im WVG eine aufgrund einer Risikobewertung angepasste Probennahmeplanung (RAP) vom Gesundheitsamt nach TrinkwV } 2001 \text { genehmigt } \\
\text { wurde. }\end{array}$} \\
\hline
\end{tabular}


Tab. A-11-1 Informationen über Änderungen der WVG in dem Land

(Informationen über Schließung, Einrichtung, Teilung oder Zusammenlegung von WVG, in denen mehr als $1000 \mathrm{~m}^{3}$ Wasser für den menschli-

chen Gebrauch pro Tag verteilt oder mehr als 5000 Personen versorgt werden.)

Landescode $^{5}$

Jahr

WVG-ID des geschlossenen WVG

Datum der Schließung ${ }^{1}$

Gründe der Schließung ${ }^{2}$

WVG-ID des neuen WVG ${ }^{3}$

Datum der Einrichtung ${ }^{4}$

${ }^{1}$ Datum (TT.MM.JJJ) an dem ein im Vorjahr berichtspflichtiges WVG geschlossen wurde.

${ }^{2}$ Gründe für die Schließung mit nachfolgenden Codes benennen. W: schlechte Wasserqualität, bei ein oder mehreren Parametern konnte der Grenzwert über längeren Zeitraum (z. B. 3 mal 3 Jahre) nicht eingehalten werden, geeignete Abhilfemaßnahmen waren nicht erfolgreich oder möglich; N: SchlieBung wegen Neufestlegung von WVG oder Zuordnung zu anderem(n) WVG; $\mathrm{O}$ : andere Gründe für geänderte Trinkwasserbereitstellung.

${ }^{3} \mathrm{Ggf}$. siehe im Anhang Beispiele für Angaben in Tab. A-11-1.

${ }^{4}$ Datum (TT.MM.JJJJ) an dem ein neues WVG eingerichtet wurde.

${ }^{5}$ Siehe Tab. A-1 Anm. 6.

Tab. A-12 Codes für den Zeitrahmen bei Einschränkung oder Unterbrechung der Bereitstellung von Wasser für den menschlichen Gebrauch (zu Tab. A4-1)

\begin{tabular}{ll} 
Code & Zeitrahmen \\
\hline I & Umgehend, d.h. nicht mehr als 1 Tag \\
V & Sehr kurzfristig, d.h. nicht mehr als 1 Woche \\
S & Kurzfristig, d.h. nicht mehr als 30 Tage \\
M & Mittelfristig, d.h. mehr als 30 Tage, aber nicht mehr als ein Jahr \\
L & Langfristig, d.h. mehr als ein Jahr
\end{tabular}

\section{Tab. A-13 Codes für die Ursachen einer Nichteinhaltung (zu Tab. A-5)}

\section{Code Ursache}

C auf Einzugsgebiet zurückzuführen

T auf Wasserwerk zurückzuführen

P auf öffentliches Verteilungsnetz zurückzuführen

D auf häusliche Trinkwasser-Installation zurückzuführen

O Andere Ursache

S Kombination an Ursachen

U Unbekannte Ursache 


\section{Tab. A-14 Codes für Abhilfemaßnahmen bei Nichteinhaltung (zu Tab. A-6)}

\begin{tabular}{|c|c|}
\hline Code & Beschreibung der Abhilfemaßnahme \\
\hline \multicolumn{2}{|c|}{ C für Einzugsgebiet } \\
\hline C1 & Maßnahme(n) zur Behebung bzw. Minderung der Ursache (einschließlich Verschneiden der Wässer aus verschiedenen Brunnen) \\
\hline $\mathrm{C} 2$ & Maßnahme(n) zum Ersatz der Wasserressource \\
\hline \multicolumn{2}{|c|}{ Tfür Aufbereitung } \\
\hline $\mathrm{T} 1$ & Einführung, Aufrüstung oder Ertüchtigung der Aufbereitung \\
\hline \multicolumn{2}{|c|}{ P für öffentliches Verteilungsnetz } \\
\hline P1 & Austausch, Abtrennung oder Reparatur defekter Teile \\
\hline P2 & Mechanische/chemische Reinigung und/oder Desinfektion verunreinigter Teile \\
\hline \multicolumn{2}{|c|}{ D für Trinkwasser-Installation ${ }^{1}$} \\
\hline D1 & Austausch, Abtrennung oder Reparatur defekter Teile \\
\hline D2 & Mechanische/chemische Reinigung und/oder Desinfektion verunreinigter Teile \\
\hline \multicolumn{2}{|c|}{ S Sicherungsmaßnahmen, um Zutritt durch Unbefugte zu verhindern } \\
\hline S1 & Sicherungsmaßnahmen, um Zutritt durch Unbefugte zu verhindern \\
\hline \multicolumn{2}{|c|}{ OSonstiges } \\
\hline 01 & Sonstiges \\
\hline \multicolumn{2}{|c|}{ E Notfallmaßnahmen im Sinne der Verbrauchergesundheit und -sicherheit } \\
\hline E1 & Benachrichtigung der Verbraucher und Anweisungen (z. B. Entnahmeverbot, Abkochgebot, vorübergehende Gebrauchseinschränkung) \\
\hline E2 & Vorübergehende Bereitstellung einer alternativen Trinkwasserversorgung (z. B. Wasser in Flaschen, Behältern, Tankwagen) \\
\hline \multicolumn{2}{|c|}{ R Kein Handeln erforderlich } \\
\hline R1 & Kein Handeln im Sinne von §9 Absatz 5a TrinkwV 2001 unter dem Gesichtspunkt des Strahlenschutzes erforderlich \\
\hline
\end{tabular}

\section{Tab. A-15 Codes für den Zeitrahmen der Abhilfemaßnahmen (zu Tab. A-6)}

\section{Code Zeitrahmen}

I Umgehend, d.h. nicht mehr als 1 Tag

S Kurzfristig, d.h. nicht mehr als 30 Tage

M Mittelfristig, d.h. mehr als 30 Tage, aber nicht mehr als ein Jahr

L L Langfristig, d.h. mehr als ein Jahr

\section{Berichterstattung gemäß Trinkwasserverordnung ${ }^{4}$}

\subsection{Rechtliche Grundlage und allgemeine Anforderungen}

Gemäß $₫ 21$ Absatz 3 TrinkwV 2001 berichten die zuständigen obersten Landesbehörden nach den Formatvorgaben der EU-Kommission über die Trinkwasserbeschaffenheit in Wasserversorgungsgebieten (WVG), in denen mindestens $10 \mathrm{~m}^{3}$

\footnotetext{
${ }^{4}$ Hier werden die WVG berücksichtigt, über die nicht bereits nach TW-RL zu berichten ist. Nicht zu berücksichtigen sind WVG, in denen weniger als $10 \mathrm{~m}^{3}$ Trinkwasser pro Tag verteilt und weniger als 50 Personen versorgt werden.
}

Wasser pro Tag geliefert oder mindestens 50 Personen versorgt werden. Dies verlangt, die Mindestanforderungen in der Trinkwasserberichterstattung auf kleinere als in der TW-RL festgelegte WVG anzuwenden. Bund und Länder haben sich darauf verständigt, für die WVG mit dem TrinkwV-spezifischen Größenbereich aus praktischen Erwägungen ein separates Formblatt zu verwenden.

Die Einbeziehung der Parameter Radon, Tritium und Richtdosis in die Überwachung der Trinkwasserqualität bedingt, die erforderlichen Angaben zu den Parametern Tritium und Richtdosis unter Beachtung der Dritten Verordnung zur Änderung der Trinkwasserverordnung [1] im bisherigen nationalen Berichts- format zu konkretisieren und den Parameter Radon $\mathrm{zu}$ integrieren (siehe hierzu auch in Kap. 2). Untersuchungen von Wasserversorgungsanlagen gemäß $\$ 3$ Nummer 2 Buchstabe b TrinkwV 2001 sind nur erforderlich, wenn die zuständige Behörde dies anordnet ( $\$ 14 \mathrm{a}$ Absatz 1 TrinkwV 2001).

Das Format übernimmt im Wesentlichen die Vorgaben der Berichterstattung nach TW-RL. Datenerfassung und Datenbereitstellung müssen den Vorgaben der EU-Kommission für die europäische Berichtspflichten genügen, weil auch mehr 
Tab. B-1 Jahresübersicht über WVG ${ }^{7}$, in denen die Überwachungshäufigkeit nicht eingehalten wird ${ }^{2}$

(Informationen zur Anzahl der durchgeführten Untersuchungen verglichen mit der Anzahl der erforderlichen Untersuchungen)²

Landescode $^{6}$

Jahr

$\begin{array}{lllll}\text { Bezeichnung des WVG' } & \text { WVG-ID } & \text { Betroffener Parameter } & \begin{array}{l}\text { Geforderte Anzahl der } \\ \text { Untersuchungen }\end{array} & \begin{array}{l}\text { Anzahl der durchgeführten } \\ \text { Untersuchungen }^{3,4}\end{array}\end{array}$

1 aus Tab. B-11.

${ }^{2}$ Umfang und Häufigkeit der Untersuchungen bestimmen sich nach TrinkwV 2001. Die genannte Anzahl der Untersuchungen muss die ausgeschöpften Möglichkeiten der Reduzierung nach TrinkwV 2001 beinhalten [vgl. §19 Abs. 2 Satz 2 Nr. 1 und Anlage 4 Teil I Buchstabe a Satz 2 und 3 und Buchstabe b Satz 1 und 2 TrinkwV 2001 und Hinweise in Anlage 3 Teil I Ifd. Nr. 4 TrinkwV 2001 (zu Clostridium perfringens), in Anlage 3 Teil I Ifd. Nr. 16 TrinkwV 2001 (zu Oxidierbarkeit)]. Die geforderte Mindestanzahl an Untersuchungen ist in einem Probennahmeplan festzuhalten. Etwaige zusätzliche Untersuchungen, die notwendig werden, um die Einhaltung zugelassener Abweichungen vom Grenzwert nach § 10 TrinkwV 2001 zu überwachen, sind nicht der geforderten Anzahl der Untersuchungen zuzurechnen, die sich aus Anlage 4 Teil II Buchstabe a ableitet. In die Anzahl der Untersuchungen gehen auch jene Untersuchungen zur Betriebskontrolle nicht ein, die der Betreiber zusätzlich zu der vom Gesundheitsamt geforderten Mindestanzahl an Proben pro Jahr durchführt.

${ }^{3}$ Dies gilt auch für die Anforderung, den Parameterwert für Nitrit am Ausgang des Wasserwerks sicherzustellen (Anlage 2 Teil II Bemerkung in Ifd. Nr. 9 TrinkwV 2001).

${ }^{4}$ In die Anzahl der Untersuchungen können jene nach §19 Abs. 7 TrinkwV 2001 (Überwachung in häuslichen Trinkwasser-Installationen) mit eingehen.

${ }^{5}$ Für die Parameter Radon und Richtdosis gelten die Mindesthäufigkeiten der Untersuchungen nach Anlage 3a Teil III Nr. 1 TrinkwV 2001 in Verbindung mit § 14a und § 20a Abs. 3 TrinkwV 2001; für den Parameter Tritium gilt ggf. die Anordnung der zuständigen Behörde nach §14a Abs. 1 Satz 5 TrinkwV 2001.

${ }^{6}$ Brandenburg: DEBB, Berlin: DEBE, Baden-Württemberg: DEBW, Bayern: DEBY, Bremen: DEHB, Hamburg: DEHH, Hessen: DEHE, Mecklenburg-Vorpommern: DEMV, Niedersachsen: DENI, Nordrhein-Westfalen: DENW, Rheinland-Pfalz: DERP, Schleswig-Holstein: DESH, Saarland: DESL, Sachsen: DESN, Sachsen-Anhalt: DEST, Thüringen: DETH.

${ }^{7} \mathrm{WVG}$, in denen mindestens $10 \mathrm{~m}^{3}$ Trinkwasser pro Tag verteilt oder mindestens 50 Personen versorgt werden sowie höchstens $1000 \mathrm{~m}^{3}$ Trinkwasser pro Tag verteilt und höchstens 5000 Personen versorgt werden.

und mehr die „kleinen“ $W V G^{5}$ in die routinemäßige Berichterstattung an die EUKommission einbezogen werden. Das Berichtsformat legt dafür die Grenzwerte und Anforderungen der TrinkwV 2001 zugrunde und berücksichtigt die aus der TrinkwV 2001 resultierenden Besonderheiten. Die oben genannten Grundsätze 2-1 bis 2-11 in der BMG-Mitteilung von 2008 [2] gelten entsprechend mit der Einschränkung, dass

- die Landesbehörden an das UBA jährlich über die Beschaffenheit von Trinkwasser aus WVG berichten, in denen im Durchschnitt mindestens $10 \mathrm{~m}^{3}$ Wasser pro Tag verteilt oder mindestens 50 Personen versorgt werden sowie höchstens $1000 \mathrm{~m}^{3}$ Wasser pro Tag verteilt und höchstens 5000 Personen versorgt werden, und

- für die Mitteilungen und Informationen über zugelassene Abweichungen die Festlegungen in $\$ 10$ TrinkwV 2001 gelten, Angaben aus diesen Mit-

\footnotetext{
${ }^{5}$ WVG, in denen mindestens $10 \mathrm{~m}^{3}$ Trinkwasser pro Tag verteilt oder mindestens 50 Personen versorgt werden sowie höchstens $1000 \mathrm{~m}^{3}$ Trinkwasser pro Tag verteilt und höchstens 5000 Personen versorgt werden
}

teilungen und Informationen auch Bestandteil der Berichte über die Trinkwasserbeschaffenheit in WVG mit TrinkwV-spezifischem Größenbereich sind und den Mitteilungen und Informationen nach $\$ 10$ TrinkwV 2001 die in Anlage 2 TrinkwV 2001 genannten Grenzwerte zugrunde liegen.

Die obersten Landesbehörden berichten dem UBA ab dem Berichtsjahr 2020 nach dem hier vorgegeben Format in der in $\$ 21$ Absatz 3 TrinkwV 2001 genannten Frist. Über die Parameter der Radioaktivität in Wasserversorgungsgebieten mit bestehenden Wasserversorgungsanlagen berichten die obersten Landesbehörden unter Beachtung des $\$ 14 \mathrm{a}$ Abs. 2 Satz 2 in Verbindung mit der Anlage 3a Teil III ebenfalls ab dem Berichtsjahr 2020. Für neu in Betrieb genommene Wasserversorgungsanlagen ist die Erstuntersuchung innerhalb der auf die Inbetriebnahme folgenden 12 Monate abzuschließen und der Bericht in das darauf folgende Berichtsjahr aufzunehmen.

Der Bericht ist als XML-Datei über die für Trinkwasser spezifizierte XML-Schnittstelle in die zentrale Datenhaltung des „WasserBLIcK“ bei der BfG einzustellen.

\subsection{Informationen, die dem Umweltbundesamt jährlich vorzulegen sind}

\subsubsection{Informationen zur Einhaltung der Mindestüberwachungshäufigkeit in Wasserversorgungsgebieten}

Diese Informationen sind nach dem Format der $\bullet$ Tab. B-1 bereitzustellen. ${ }^{6}$ Untersuchungen der Parameter Radon und Richtdosis sind zu berücksichtigen, wenn die zuständige Behörde keine anderweitige Feststellung nach $\$ 14 \mathrm{a}$ Abs. 4 TrinkwV 2001 getroffen hat. Untersuchungen des Parameters Tritium sind zu berücksichtigen, wenn die zuständige Behörde diese nach $₫ 14$ a Abs. 1 Satz 5 TrinkwV 2001 angeordnet hat.

\footnotetext{
${ }^{6}$ Die Tabellennummerierung folgt dem Guidance document (siehe Fußnote 1). Der hinzugefügte Großbuchstabe dient der besseren Zuordnung der im nationalen Format verwendeten Tabellen. Nachfolgend ist die Tabellennummer B-7 nicht vergeben, da im Guidance document eine Tabellennummer 7 fehlt bzw. gestrichen worden ist, ohne die Tabellenreihung daran anzupassen.
} 
Tab. B-2 Allgemeine Informationen über die Rahmenbedingungen der Trinkwasserversorgung in dem Land

(Mit Ausnahme von Frage 1.2 werden nur Informationen über WVG gemeldet, in denen mindestens $10 \mathrm{~m}^{3}$ Trinkwasser pro Tag verteilt oder

mindestens 50 Personen versorgt werden sowie höchstens $1000 \mathrm{~m}^{3}$ Trinkwasser pro Tag verteilt und höchstens 5000 Personen versorgt wer-

den.)

\begin{tabular}{|c|c|c|}
\hline 1.1 .1 & Landescode $^{6}$ & \\
\hline 1.1.2. & Jahr & \\
\hline 1.2 & Gesamtbevölkerung in Mio. ${ }^{1}$ & \\
\hline 1.3 & Anzahl der WVG & \\
\hline 1.4 & Gesamte in den WVG versorgte Bevölkerung ${ }^{2}$ & \\
\hline 1.5 & Gesamtes in den WVG verteiltes Wasservolumen in Mio. $\mathrm{m}^{3} / \mathrm{Jahr}$ & \\
\hline 1.6 & Für 1.5 genutzte Wasserressourcen (=100\%) & \\
\hline 1.6 .1 & Anteil von 1.6 an Grundwasser ${ }^{3}$ in Prozent & \\
\hline 1.6 .2 & Anteil von 1.6 an Oberflächenwasser ${ }^{4}$ in Prozent & \\
\hline 1.6.2.1 & Anteil von 1.6 an Binnenwasser in Prozent & \\
\hline 1.6.2.2 & Anteil von 1.6 an Küsten-, Übergangs-, (Brack)wasser in Prozent & \\
\hline 1.6 .3 & Anteil von 1.6 an Uferfiltrat in Prozent & \\
\hline 1.6 .4 & Anteil von 1.6 an künstlicher Grundwasseranreicherung in Prozent & \\
\hline 1.6 .5 & Anteil von 1.6 an Regenwasser ${ }^{5}$ in Prozent & \\
\hline 1.6 .6 & Anteil von 1.6 an sonstigen Ressourcen in Prozent & \\
\hline 1.7 & Landesdatenbank zur Qualität von Wasser für den menschlichen Gebrauch & \\
\hline 1.7 .1 & Öffentlicher Zugang zur Datenbank unter folgender Webseiten-Adresse & \\
\hline 1.8 & Kontaktstelle im Land & \\
\hline 1.8 .1 & Zuständige Behörde & \\
\hline 1.8 .2 & Ansprechpartner & \\
\hline 1.8 .3 & Anschrift & \\
\hline 1.8 .4 & Telefon & \\
\hline 1.8 .5 & Fax & \\
\hline 1.8.6 & E-Mail & \\
\hline \multicolumn{3}{|c|}{$\begin{array}{l}\text { Anmerkungen: } \\
{ }^{1} \text { Die Gesamtbevölkerung in Mio. soll bis zu drei Stellen nach dem Komma angegeben werden. } \\
\text { 2Die Bevölkerung in } 1.4 \text { wird als ganze Zahl (nicht durch Dezimalbezeichnungen abgekürzt) gemeldet. } \\
{ }^{3} \text { Grundwasser bedeutet alles unterirdische Wasser in der Sättigungszone, das in unmittelbarer Berührung mit dem Boden oder dem Untergrund steht. } \\
{ }^{4} \text { Oberflächenwasser bedeutet Binnenwasser (ohne Grundwasser), Übergangswasser und Küstenwasser (Wasserrahmenrichtlinie 2000/60/EG Art. 2.1). } \\
\text { 5 Regenwasser bedeutet hier nur Regenwasser, das ausschließlich als Ressource für die Trinkwasserversorgung genutzt wird. Hierzu gehört nicht Dachablaufwasser für } \\
\text { häusliche Zwecke. } \\
{ }^{6} \text { Siehe Tab. B-1 Anm. } 6 \text {. }\end{array}$} \\
\hline
\end{tabular}

\subsubsection{Informationen über die} allgemeinen Rahmenbedingungen zur Trinkwasserversorgung im jeweiligen Land

Das erforderliche Format ist durch

- Tab. B-2, B-11 und B-11-1 bestimmt.

\subsubsection{Informationen zur \\ Trinkwasserqualität in Wasserversorgungsgebieten auf landesweiter Ebene}

Diese Informationen sind in dem Format gemäß Tab. B-3 wiederzugeben (Raum für weitere, auch fakultative Angaben zu

- Tab. B-3 bietet - Tab. B-3-2).

3.2.4 Information über die Ergebnisse der Nachforschungen bei Nichteinhaltung des Parameterwertes Clostridium perfringens

Dafür ist - Tab. B-3-1 heranzuziehen.

\subsubsection{Informationen zu Nichteinhaltungen von Parameterwerten in den betroffenen und in - Tab. B-11 bezeichneten Wasserversorgungsgebieten}

Diese Informationen müssen den Vorgaben gemäß - Tab. B-4, B-4-1, B-5 und B-6 entsprechen. Hier sind auch jene von Artikel 9 TW-RL erfassten zugelassenen Abweichungen im Berichtsjahr zu berücksichtigen, über die das UBA bereits nach $\$ 10$ TrinkwV 2001 von den zuständigen obersten Landesbehörden unterrichtet 


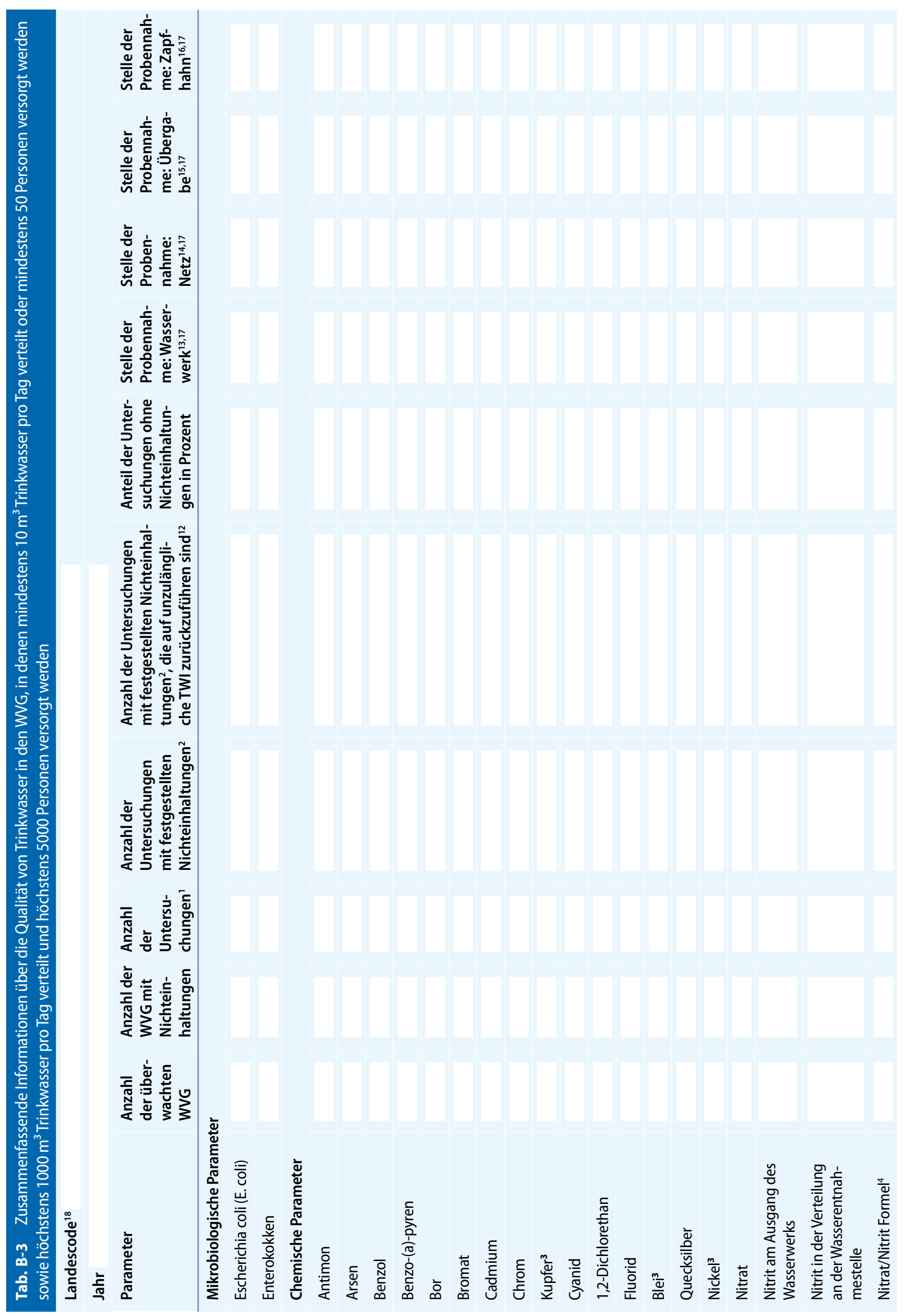




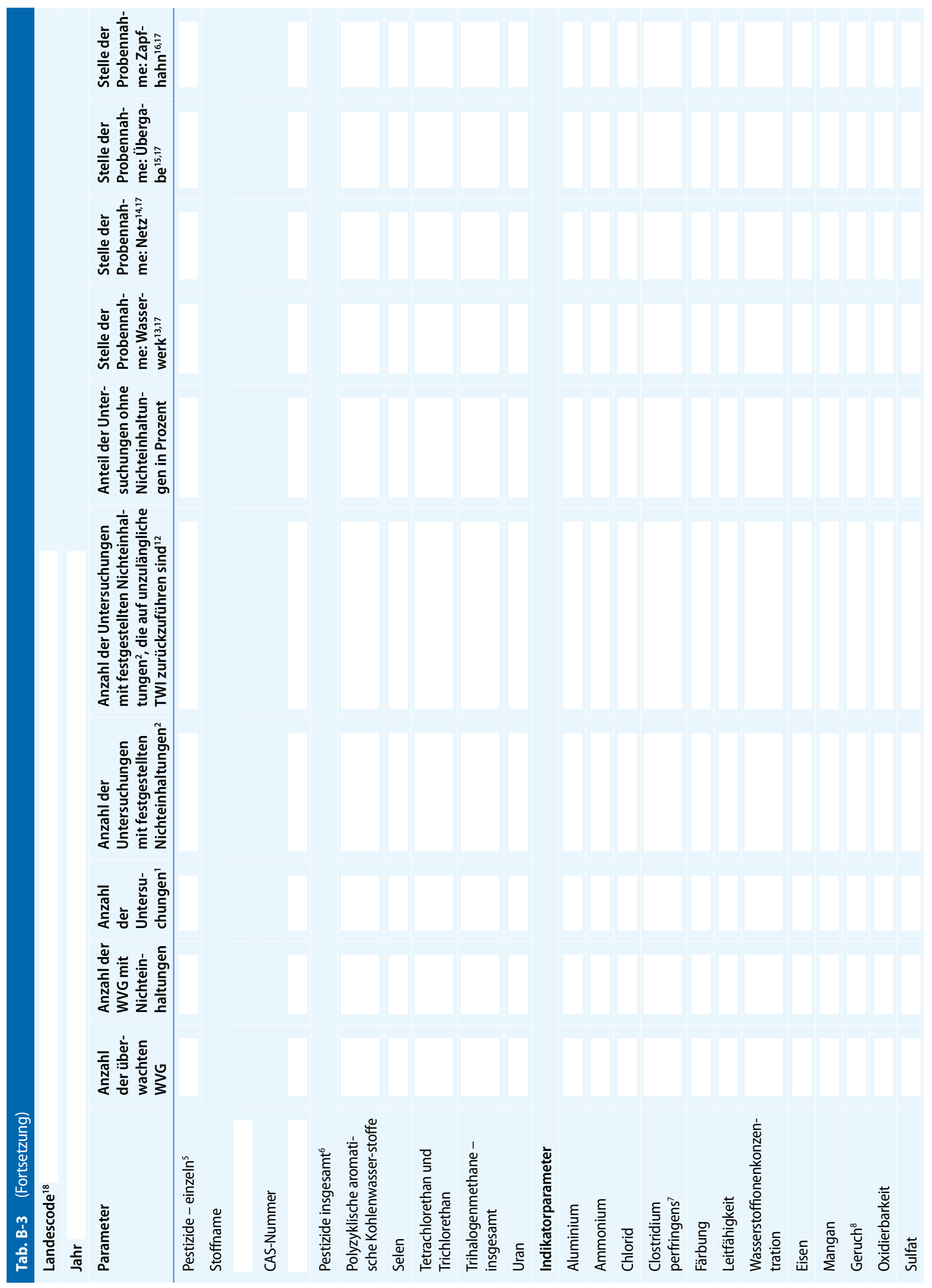




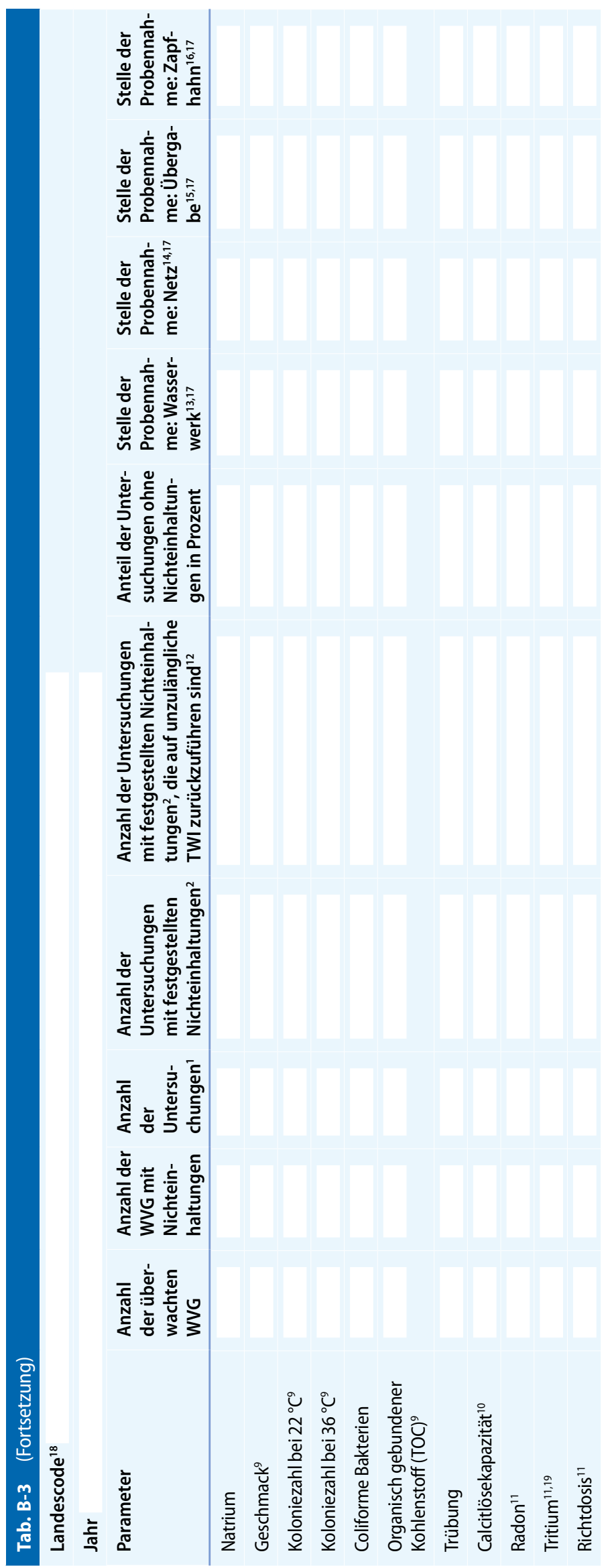

914 Bundesgesundheitsblatt - Gesundheitsforschung - Gesundheitsschutz 8 2017 


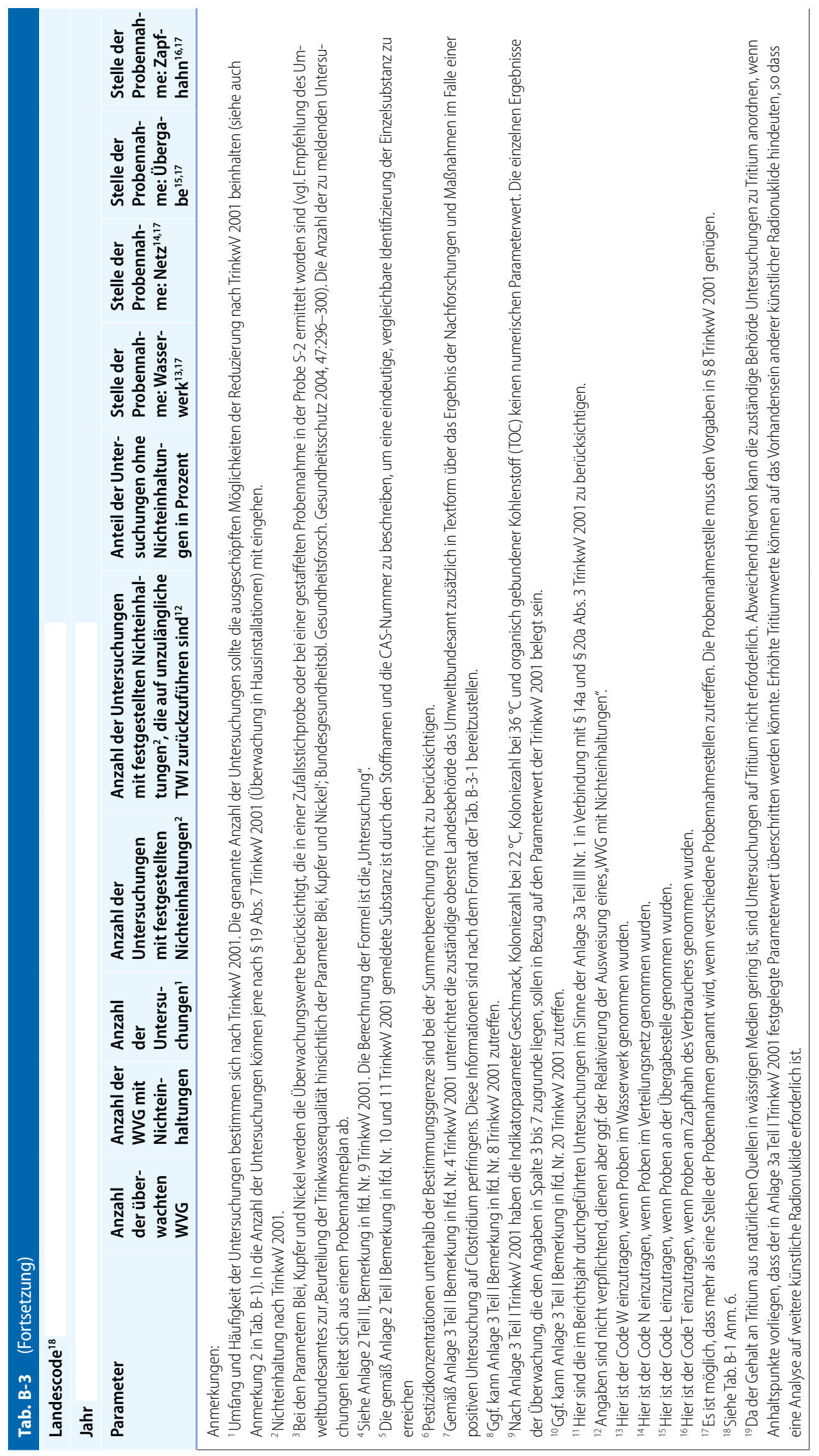


Tab. B-3-1 Information über die Ergebnisse der Nachforschungen bei Nichteinhaltung des Parameterwertes Clostridium perfringens

(einschließlich Sporen) im WVG7 gemäß Anlage 3 lfd. Nr. 4 TrinkwV 2001,2

Landescode ${ }^{6}$

Jahr

WVG $^{3}$

WVG-ID

Datum der ersten Feststellung der Nichteinhaltung

Zeitspanne bis zur Beseitigung der Nichteinhaltung (in Tagen)

Festgestellte Nichteinhaltung (Anzahl/100 ml; maximal drei Messwerte)

Zusätzliche Grenzwertüberschreitungen bei coliformen Bakterien, E. coli, Enterokokken

Ja/Nein

Ursache(n) für die Nichteinhaltung

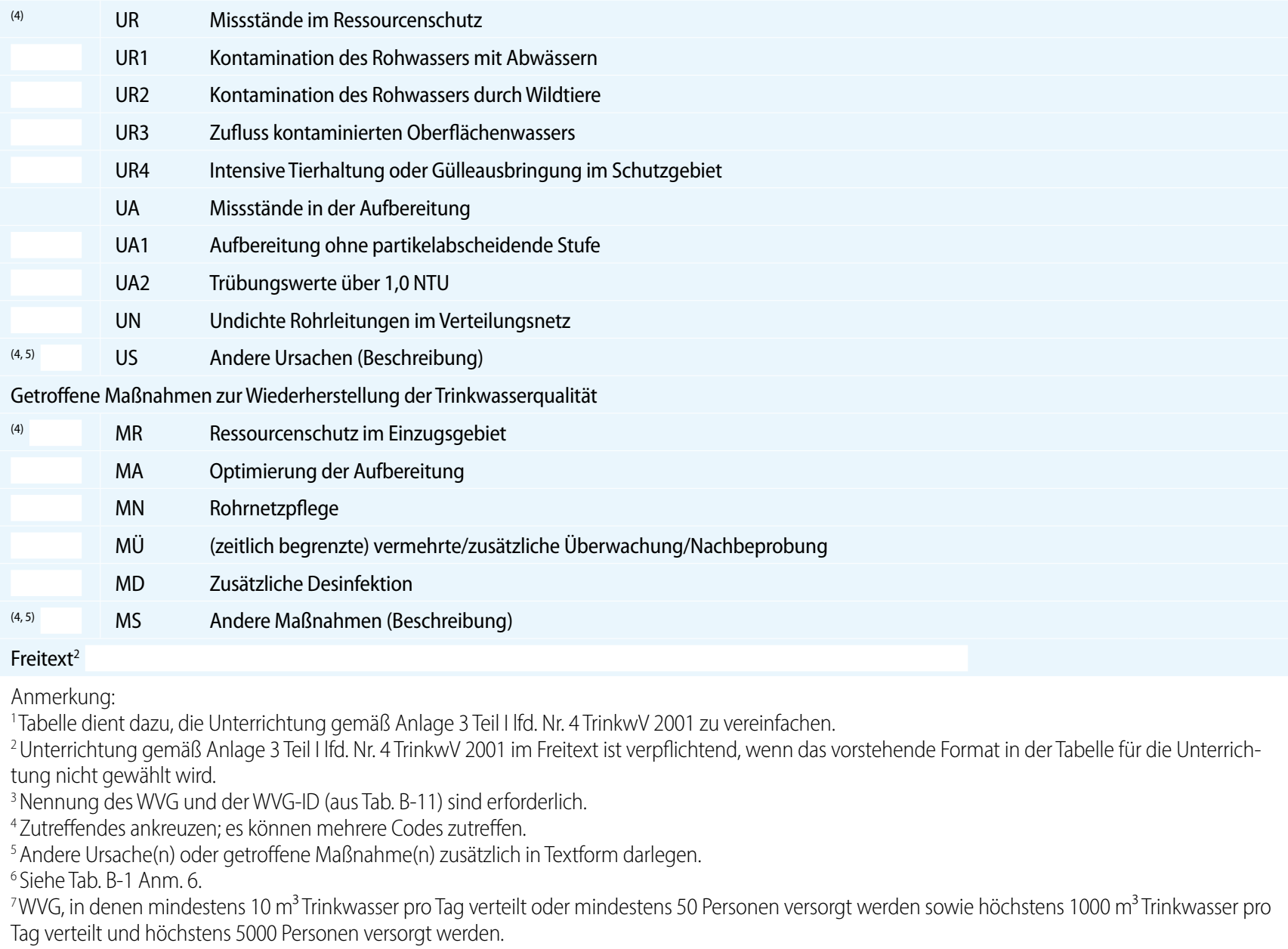

\section{Tab. B-3-2 Weitere Bemerkungen des Landes zu Tab. B-3}

Landescode ${ }^{1}$

Jahr

Freitext

' Siehe Tab. B-1 Anm. 6. 
Tab. B-4 Informationen über Nichteinhaltung der Parameterwerte bei Trinkwasser in WVG, in denen mindestens $10 \mathrm{~m}^{3}$ Trinkwasser pro Tag

verteilt oder mindestens 50 Personen versorgt werden sowie höchstens $1000 \mathrm{~m}^{3}$ Trinkwasser pro Tag verteilt und höchstens 5000 Personen

versorgt werden

Landescode ${ }^{8}$

Jahr

Parame- Ggf. Aktenzeichen

ter $^{1} \quad$ der zuständigen

obersten Landes-

behörde für die

Zulassung einer

Abweichung $^{9}$

\section{Anzahl der Gesamtzahl betroffe- der Untersu- nen Bevöl- chungen (im kerung Berichtsjahr)}

\begin{tabular}{|c|c|c|c|c|}
\hline $\begin{array}{l}\text { Gesamtzahl der } \\
\text { Untersuchun- } \\
\text { gen mit Nicht- } \\
\text { einhaltung (im } \\
\text { Berichtsjahr) }{ }^{1,2}\end{array}$ & $\operatorname{Max}^{3}$ & $\begin{array}{l}\text { Med } \\
\text { total }\end{array}$ & $\begin{array}{l}\text { Med } \\
\text { noncom- } \\
\text { plient }^{5}\end{array}$ & $\begin{array}{l}\mathrm{NCl} \\
\mathrm{ROW}- \\
\mathrm{ID}^{6,7}\end{array}$ \\
\hline
\end{tabular}

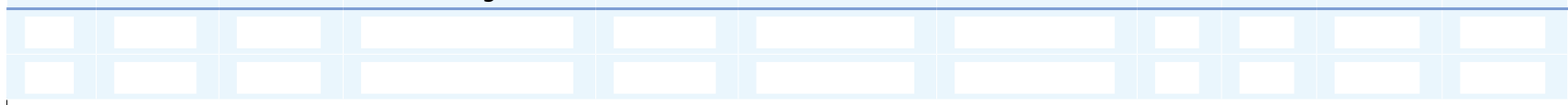

Anmerkung:

${ }^{1}$ Zu berücksichtigen sind Nichteinhaltungen vom Parameterwert jedes Parameters in Anlage 1, 2 und 3 Teil I TrinkwV 2001 bzw. zugelassene Abweichungen vom Parameterwert jedes Parameters in Anlage 2 TrinkwV 2001. Hier sind auch Überschreitungen der Parameterwerte für Radon, Tritium und die Richtdosis gemäß TrinkwV 2001 zu berücksichtigen, nachfolgende Anmerkungen gelten entsprechend.

${ }^{2}$ Zu berücksichtigen sind alle Ergebnisse, die die Parameterwerte der TrinkwV 2001 nicht erfüllen, einschließlich jener, die unter eine zugelassene Abweichung nach § 10 TrinkwV 2001 fallen (vgl. Anm. 7).

${ }^{3}$ Der Wert zeigt die Höchstkonzentration des betroffenen Parameters im Trinkwasser des WVG an, bezogen auf das Berichtsjahr oder das separate Ereignis (vgl. Anm. 7).

${ }^{4}$ Medianwert aller Untersuchungsergebnisse des betroffenen Parameters im Berichtsjahr.

${ }^{5}$ Medianwert der Untersuchungsergebnisse, die den Parameterwert der TrinkwV 2001 nicht erfüllen (vgl. auch Anm. 7).

${ }^{6}$ Hier ist der eindeutige ID-Code der Nichteinhaltung des betroffenen Parameters einzutragen.

${ }^{7}$ Tritt in einem WVG beim gleichen Parameter mehr als ein Ereignis mit Nichteinhaltung auf und lassen sich die Nichteinhaltungen auf verschiedene Ursachen zurückführen, dann werden die Ereignisse in getrennten Zeilen aufgeführt. Jede Zeile ist mit einer eigenen NCI_ROW-ID (in Spalte 11) zu kennzeichnen. Liegen mehr als ein Ereignis

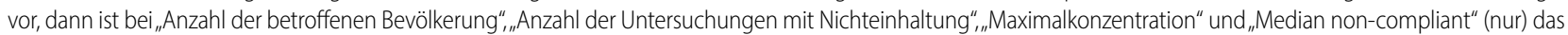
jeweilige Ereignis (nicht das gesamte Berichtsjahr) zu berücksichtigen. Die Benennung der verschiedenen Ursachen ist der Angabe Kombination an Ursachen (mit Code S in Tab. B-5) vorzuziehen. (Zeitlich versetzte) Ereignisse mit gleicher Ursache werden als ein Ereignis im Berichtsjahr betrachtet.

${ }^{8}$ Siehe Tab. B-1 Anm. 6.

${ }^{9}$ Das Az. ist einzutragen, wenn es sich auf eine Zulassung/Beantragung einer Abweichung im Sinne von $\S 10$ Abs. 5 oder 6 TrinkwV 2001 bezieht. Das Az. ist nicht erforderlich, wenn es sich um die erste Zulassung einer Abweichung im Sinne von § 10 Abs. 2 TrinkwV 2001 handelt.

Tab. B-4-1 Informationen über Einschränkung/Unterbrechung der Bereitstellung von Trinkwasser wegen Nichteinhaltung der Parameterwerte im WVG

\section{NCl_ROW-ID ${ }^{1}$ WVG-ID Parameter Einschränkung J/N² Unterbrechung J/N $\mathrm{N}^{3}$ Gründe für die Einschränkung/ Zeitrahmen (Code) $)^{5}$} Unterbrechung ${ }^{4}$

Anmerkung:

${ }^{1}$ Aus Tab. B-4 zu übernehmen, siehe Tab. B-4 Anm. 6.

2 J eintragen, wenn Wasserbereitstellung im WVG eingeschränkt war; N eintragen, wenn keine Einschränkung bestand.

3 J eintragen, wenn Wasserbereitstellung im WVG unterbrochen war; N eintragen, wenn es nicht zu einer Unterbrechung kam.

${ }^{4}$ In Textform die Gründe für die Einschränkung oder Unterbrechung der Wasserbereitstellung benennen.

${ }^{5}$ Hier ist ein Code aus Tab. B-12 einzutragen.

Tab. B-5 Informationen über Ursachen der Nichteinhaltung der Parameterwerte im WVG

\begin{tabular}{|c|c|c|c|c|c|}
\hline NCl_ROW-ID' & WVG-ID & Parameter & Ursache $^{2}$ & Anzahl der Untersuchungen mit Nichteinhaltung ${ }^{3}$ & Remedial-ID 4 \\
\hline \multicolumn{6}{|c|}{$\begin{array}{l}\text { Anmerkung: } \\
{ }^{1} \text { Aus Tab. B-4 zu übernehmen, siehe Tab. B-4 Anm. } 6 . \\
{ }^{2} \text { Hier ist ein Code aus Tab. B-13 einzutragen, siehe auch Tab. B-4 Anm. 7. Sind für die Beseitigung einer Ursache mehr als eine Abhilfemaßnahme anzugeben, dann ist der } \\
\text { einzelnen, in Tab. B-6 zu benennenden Abhilfemaßnahme bereits in Tab. B-5 eine eigene Remedial-ID (in Spalte 6) in einer separaten Zeile zuzuweisen. } \\
{ }^{3} \text { Es sind jene Ereignisse, die die Parameterwerte der TrinkwV } 2001 \text { nicht erfüllen, zu berücksichtigen, die mit dieser Ursache zusammenhängen. } \\
{ }^{4} \text { Hier ist der eindeutige ID-Code der Abhilfemaßnahmen einzutragen. }\end{array}$} \\
\hline
\end{tabular}


Tab. B-6 Informationen über Abhilfemaßnahmen bei Nichteinhaltung der Parameterwerte im WVG

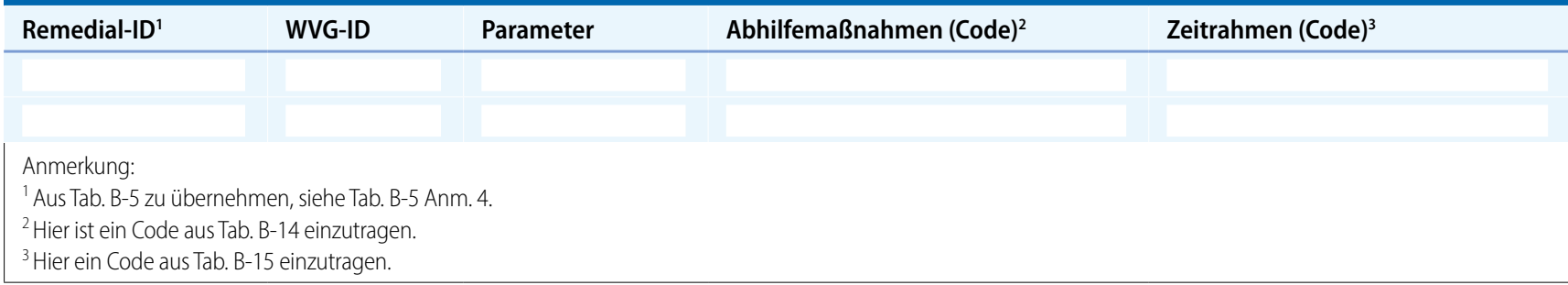

Tab. B-8 Informationen über die produktspezifischen Parameter Acrylamid, Epichlorhydrin und Vinylchlorid

Landescode ${ }^{2}$

Jahr

Parameter Erläuterungen, wie die Einhaltung der Anforderungen sichergestellt ist ${ }^{1}$

Acrylamid

Epichlorhydrin

Vinylchlorid

Anmerkung:

${ }^{1}$ Gemäß Anlage 3 Teil I Bemerkung in Ifd. Nr. 1 und Teil II Bemerkungen in Ifd. Nr. 6 und 12TrinkwV 2001 müssen die produktbezogenen Parameter Acrylamid, Epichlorhydrin und Vinylchlorid nicht im Wasser analysiert werden, dennoch muss gemäß den Produktspezifikationen des entsprechenden Polymers in Kontakt mit Trinkwasser nachgewiesen werden, dass der Parameterwert nach TrinkwV 2001 eingehalten wird. Das Land erläutert, wie es diese Anforderung der TrinkwV 2001 erfüllt hat, z. B. länderspezifische Regelungen für Bauprodukte in Kontakt mit Trinkwasser. Wird Trinkwasser auch auf Acrylamid, Epichlorhydrin oder Vinylchlorid chemisch analysiert, dann ist das in dieser Tab. zu vermerken.

${ }^{2}$ Siehe Tab. B-1 Anm. 6.

\section{Tab. B-9 Art der im Land öffentlich zugänglichen Informationen}

\section{Landescode}

Jahr

Art der verfügbaren Informationen Ja/Nein Ort der Information ${ }^{2}$

9.1 Landesweite Zusammenfassung über die Einhaltung der TrinkwV 2001

9.2 Regionale Zusammenfassung über die Einhaltung der TrinkwV 2001

9.3 Zusammenfassung der Einhaltung der TrinkwV 2001 bezogen auf das WVG

9.4 Einzelne Überwachungsergebnisse für das WVG

9.5 Überwachungsergebnisse für sehr kleine WVG (in denen im Durchschnitt weniger als $10 \mathrm{~m}^{3} /$ Tag verteilt und weniger als 50 Personen versorgt werden)

9.6 Informationen über Einhaltung strengerer nationaler Standards

9.7 Informationen über Einhaltung zusätzlicher nationaler Standards

9.8 Informationen auf der Ebene eines WVG über Nichteinhaltungen

9.9 Informationen über die Trinkwasserressource(n) im betreffenden WVG

9.10 Sonstiges

Anmerkungen:

1 Siehe Tab. A-1 Anm. 6

${ }^{2}$ Bei dem Ort sollte es sich um die Stelle handeln, an die sich die EU-Kommission wenden kann, um die jeweils aufgeführten Arten von Informationen zu erhalten. 
Tab. B-10 Kommunikation der im Land verfügbaren Informationen über die Qualität von Wasser für den menschlichen Gebrauch

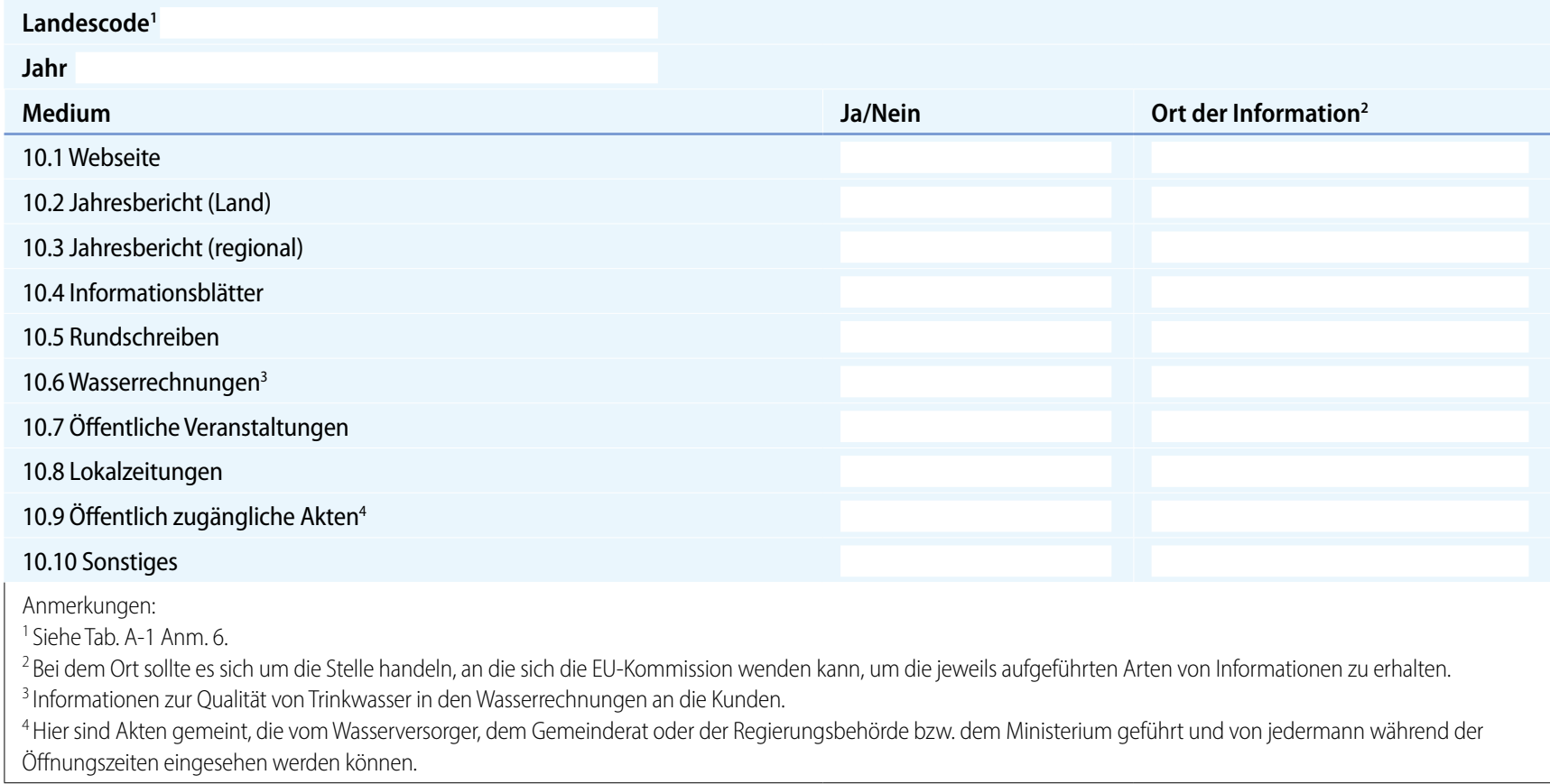

Tab. B-11 Informationen über die Wasserversorgungsgebiete in dem Land

(Informationen über WVG, in denen mindestens $10 \mathrm{~m}^{3}$ Trinkwasser pro Tag verteilt oder mindestens 50 Personen versorgt werden sowie höchstens $1000 \mathrm{~m}^{3}$ Trinkwasser pro Tag verteilt und höchstens 5000 Personen versorgt werden)

Landescode $^{6}$

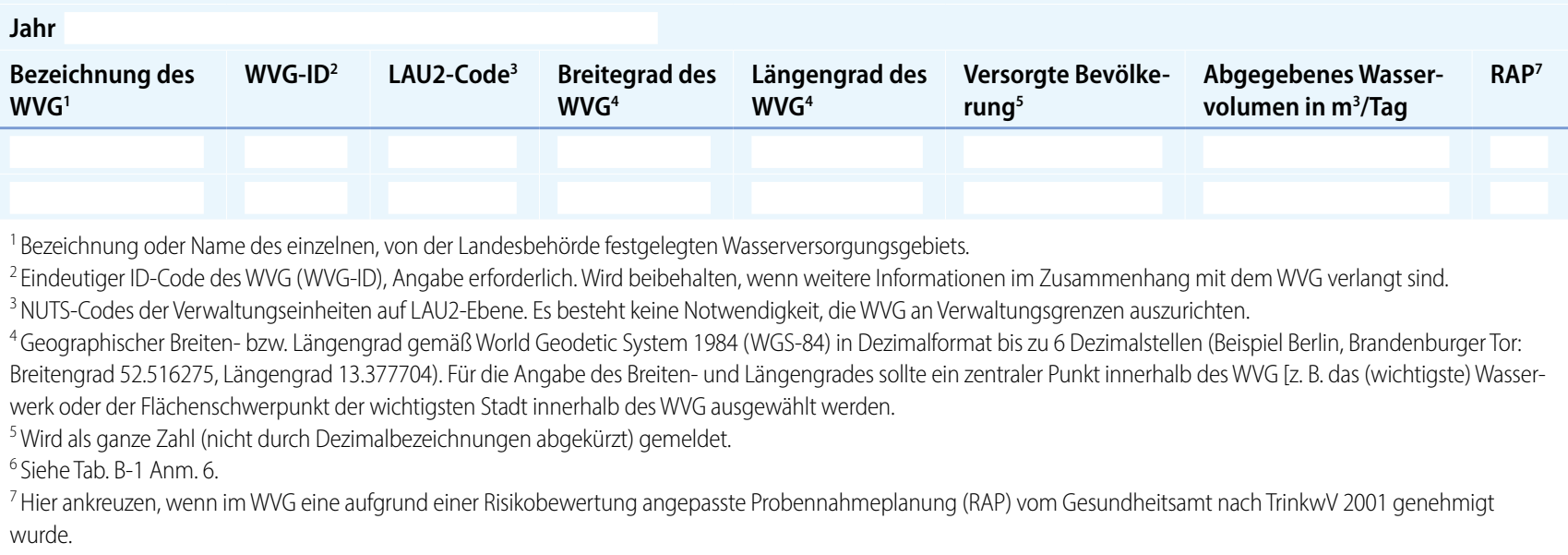

worden ist (vgl. BMG-Mitteilung 2008, Grundsatz 2-11; [2]). Die Parameter Radon, Tritium und Richtdosis sind hier ebenfalls zu berücksichtigen.

\subsubsection{Informationen über die produktspezifischen Parameter Acrylamid, Epichlorhydrin und Vinylchlorid}

(nach dem Format der • Tab. B-8)

\subsubsection{Informationen über die Qualität von Wasser für den menschlichen Gebrauch an die Öffentlichkeit}

(siehe auch BMG-Mitteilung 2008, 4. Kapitel [2])

Nach Artikel 13 Absatz 1 TW-RL sind in den Mitgliedstaaten Maßnahmen zu ergreifen, die sicherstellen, dass den Verbrauchern und Verbraucherinnen geeignetes und aktuelles Informationsmaterial über die Qualität von Wasser für den menschlichen Gebrauch zur Verfügung steht. Nach dem Format der $\bullet$ Tab. B-9 und B-10 ist zu dokumentieren, wo und in welcher Art sowohl die Öffentlichkeit als auch die EU-Kommission diese Informationen über die Trinkwasserbeschaffenheit im Land abrufen können. Die Informationen über die Trinkwasserqualität an die Öffentlichkeit sind nur dann nach dem Format der $\bullet$ Tab. B-9 und B-10 zu liefern, wenn diesbezügliche Angaben für das Berichtsjahr nicht oder nicht vollständig nach dem Format der - Tab. A-9 und A-10 bereitgestellt werden. 
Tab. B-11-1 Informationen über Änderungen der Wasserversorgungsgebiete in dem Land

(Informationen über Schließung, Einrichtung, Teilung oder Zusammenlegung von WVG, in denen mindestens $10 \mathrm{~m}^{3}$ Trinkwasser pro Tag verteilt oder mindestens 50 Personen versorgt werden sowie höchstens $1000 \mathrm{~m}^{3}$ Trinkwasser pro Tag verteilt und höchstens 5000 Personen versorgt

werden)

Landescode ${ }^{5}$

Jahr

WVG-ID des geschlossenen WVG Datum der Schließung ${ }^{1}$

Gründe der Schließung ${ }^{2} \quad$ WVG-ID des neuen WVG ${ }^{3} \quad$ Datum der Einrichtung ${ }^{4}$

${ }^{1}$ Datum (TT.MM.JJJ) an dem ein im Vorjahr berichtspflichtiges WVG geschlossen wurde.

${ }^{2}$ Gründe für die Schließung mit nachfolgenden Codes benennen. W: schlechte Wasserqualität, bei ein oder mehreren Parametern konnte der Grenzwert über längeren Zeitraum (z. B. nach 3 mal 3 Jahren) nicht eingehalten werden, geeignete Abhilfemaßnahmen waren nicht erfolgreich oder möglich; N: Schließung wegen Neufestlegung von WVG oder Zuordnung zu anderem(n) WVG; O: andere Gründe für geänderte Trinkwasserbereitstellung.

${ }^{3}$ Ggf. siehe im Anhang Beispiele für Angaben in Tab. B-11-1.

${ }^{4}$ Datum (TT.MM.JJJ) an dem ein neues WVG eingerichtet wurde.

${ }^{5}$ Siehe Tab. B-1 Anm. 6.

\section{Tab. B-12 Codes für den Zeitrahmen bei Einschränkung oder Unterbrechung der Bereitstellung von Trinkwasser (zu Tab. B4-1)}

\section{Code Zeitrahmen}

I Umgehend, d.h. nicht mehr als 1 Tag

V Sehr kurzfristig, d.h. nicht mehr als 1 Woche

S Kurzfristig, d.h. nicht mehr als 30 Tage

M Mittelfristig, d.h. mehr als 30 Tage, aber nicht mehr als ein Jahr

L $\quad$ Langfristig, d.h. mehr als ein Jahr

\section{Tab. B-13 Codes für die Ursachen einer Nichteinhaltung (zu Tab. B-5)}

\begin{tabular}{ll} 
Code & Ursache \\
\hline C & auf Einzugsgebiet zurückzuführen \\
T & auf Wasserwerk zurückzuführen \\
P & auf öffentliches Verteilungsnetz zurückzuführen \\
D & auf häusliche Trinkwasser-Installation zurückzuführen \\
O & Andere Ursache \\
S & Kombination an Ursachen \\
U & Unbekannte Ursache
\end{tabular}




\section{Tab. B-14 Codes für Abhilfemaßnahmen bei Nichteinhaltung (zu Tab. B-6)}

\section{Code Beschreibung der Abhilfemaßnahme}

C für Einzugsgebiet

C1 Maßnahme(n) zur Behebung bzw. Minderung der Ursache (einschließlich Verschneiden der Wässer aus verschiedenen Brunnen)

C2 Maßnahme(n) zum Ersatz der Wasserressource

Tfür Aufbereitung

T1 Einführung, Aufrüstung oder Ertüchtigung der Aufbereitung

P für öffentliches Verteilungsnetz

P1 Austausch, Abtrennung oder Reparatur defekter Teile

P2 Mechanische/chemische Reinigung und/oder Desinfektion verunreinigter Teile

D für Trinkwasser-Installation ${ }^{1}$

D1 Austausch, Abtrennung oder Reparatur defekter Teile

D2 Mechanische/chemische Reinigung und/oder Desinfektion verunreinigter Teile

SSicherungsmaßnahmen, um Zutritt durch Unbefugte zu verhindern

S1 Sicherungsmaßnahmen, um Zutritt durch Unbefugte zu verhindern

OSonstiges

$01 \quad$ Sonstiges

E Notfallmaßnahmen im Sinne der Verbrauchergesundheit und -sicherheit

E1 Benachrichtigung der Verbraucher und Anweisungen (z. B. Entnahmeverbot, Abkochgebot, vorübergehende Gebrauchseinschränkung)

E2 Vorübergehende Bereitstellung einer alternativen Trinkwasserversorgung (z. B. Wasser in Flaschen, Behältern, Tankwagen)

R Kein Handeln erforderlich

R1 Kein Handeln im Sinne von §9 Absatz 5a TrinkwV 2001 unter dem Gesichtspunkt des Strahlenschutzes erforderlich

'Einschließlich häuslicher Trinkwasser-Installationen, aus denen Wasser an die Öffentlichkeit abgegeben wird.

Tab. B-15 Codes für den Zeitrahmen der Abhilfemaßnahmen (zu Tab. B-6)

\begin{tabular}{ll} 
Code & Zeitrahmen \\
\hline I & Umgehend, d.h. nicht mehr als 1 Tag \\
S & Kurzfristig, d.h. nicht mehr als 30 Tage \\
M & Mittelfristig, d.h. mehr als 30 Tage, aber nicht mehr als ein Jahr \\
L & Langfristig, d.h. mehr als ein Jahr
\end{tabular}

\section{Literatur}

1. Trinkwasserverordnung (TrinkwV 2001) in der Bekanntmachung der Neufassung vom 10. März 2016, BGBI. I S. 459.

2. BMG (2008) Format für die Berichterstattung der zuständigen obersten Landesbehörden an das Bundesministerium für Gesundheit/Umweltbundesamt. Bundesgesundheitsbl Gesundheitsforsch Gesundheitsschutz 51:1078-1092

3. BMG (2013) Format für die Berichterstattung der zuständigen obersten Landesbehörden an das Bundesministerium für Gesundheit/Umweltbundesamt. Bundesgesundheitsbl Gesundheitsforsch Gesundheitsschutz 56:1191-1215

4. EU-Kommission (2011) Guidance document on reporting under the Drinking Water Directive 98/83/EC. http://icm.eionet.europa.eu/schmas/ dir199883ec/resources. Zugegriffen: 17. Juni 2017 


\section{Anhang zu ๑ Tab. A-4-1 oder B-4-1 (informativ): Beispiele}

\begin{tabular}{|c|c|c|c|c|}
\hline Landescode & DEXX & & & \\
\hline Jahr & 2014 & & & \\
\hline $\begin{array}{l}\text { WVG-ID des geschlossenen } \\
\text { WVG }\end{array}$ & Datum der Schließung & Gründe der Schließung & WVG-ID des neuen WVG & Datum der Einrichtung \\
\hline \multicolumn{5}{|c|}{ Beispiel „,Schließung und Einrichtung“ } \\
\hline DExx-15375-19 & 15.01 .2014 & $\mathrm{~N}$ & DExx-15375-1 & 19.01.2014 \\
\hline \multicolumn{5}{|l|}{ Beispiel „Teilung“ } \\
\hline DExx-15375-19 & 15.01 .2014 & $\mathrm{~N}$ & DExx-15375-1 & 19.01.2014 \\
\hline DExx-15375-19 & 15.01 .2014 & $\mathrm{~N}$ & DExx-15375-2 & 27.01 .2014 \\
\hline DExx-15375-19 & 15.01 .2014 & $\mathrm{~N}$ & DExx-15375-3 & 29.01.2014 \\
\hline \multicolumn{5}{|l|}{ Beispiel „Zusammenlegung“ } \\
\hline DExx-15375-19 & 15.01 .2014 & $\mathrm{~N}$ & DExx-15375-1 & 19.01.2014 \\
\hline DExx-15375-20 & 15.01 .2014 & $\mathrm{~N}$ & DExx-15375-1 & 19.01.2014 \\
\hline DExx-15375-21 & 15.01 .2014 & $\mathrm{~N}$ & DExx-15375-1 & 29.01 .2014 \\
\hline
\end{tabular}

Anhang zu $\bullet$ Tab. A-1 bis A-11-1 und - Tab. B-1 bis B-11-1 (informativ)

\begin{tabular}{|c|c|c|c|c|c|c|}
\hline \multirow[t]{2}{*}{ Art der Information } & \multirow[t]{2}{*}{$\begin{array}{l}\text { Ggf. Bezug } \\
\text { TrinkwV } 2001\end{array}$} & \multirow[t]{2}{*}{$\begin{array}{l}\text { Bezug Tabelle/ } \\
\text { Formblatt }\end{array}$} & \multicolumn{3}{|c|}{$\begin{array}{l}\text { Art der nach TW-RL oder TrinkwV } \\
2001 \text { betroffenen Parameter }{ }^{1}\end{array}$} & \multirow[t]{2}{*}{$\begin{array}{l}\text { Periodizität/ } \\
\text { Fristen }\end{array}$} \\
\hline & & & M & C & I & \\
\hline $\begin{array}{l}\text { Jahresübersicht zur Einhaltung der Über- } \\
\text { wachungshäufigkeit im WVG }\end{array}$ & $\begin{array}{l}\text { §14 Abs. } 2 \\
\text { §14a Abs. } 2 \\
\text { Anlage 4 Teil I Abs. } 1 \\
\text { und } 2 \\
\text { Anlage 4 Teil II } \\
\text { Anlage 3a Teil III }\end{array}$ & Tab. A-1 oder B-1 & + & + & + & Jährlich \\
\hline $\begin{array}{l}\text { Landesweite zusammenfassende Informati- } \\
\text { onen zur Qualität von Trinkwasser in WVG }\end{array}$ & & $\begin{array}{l}\text { Tab. A-3, ggf. A-3-1, } \\
\text { A-8 oder B-3, ggf. } \\
\text { B-3-1, B-8 }\end{array}$ & + & + & + & Jährlich \\
\hline $\begin{array}{l}\text { Informationen zu Nichteinhaltungen der } \\
\text { Parameterwerte bei Trinkwasser in WVG }\end{array}$ & & $\begin{array}{l}\text { Tab. A-4, A-4-1, A-5, } \\
\text { A-6 oder B-4, B-4-1, } \\
\text { B-5, B-6 }\end{array}$ & + & + & + & Jährlich \\
\hline $\begin{array}{l}\text { Art der im Land öffentlich zugänglichen } \\
\text { Informationen }\end{array}$ & $\S 21$ Abs. 1 & Tab. A-9 oder B-9 & & & & Jährlich \\
\hline
\end{tabular}

